\title{
Development of a Fixed Repertoire of Blood Transcriptome Modules Based on Co-expression Patterns Across Immunological States
}

\author{
Matthew Altman \\ Benaroya Research Institute
}

Darawan Rinchai

Sidra Medicine https://orcid.org/0000-0001-8851-7730

Nicole Baldwin

Baylor Institute for Immunology Research https://orcid.org/0000-0002-4196-3554

Mohammed Toufiq

Sidra Medicine https://orcid.org/0000-0002-6368-6746

Elizabeth Whalen

Benaroya Research Institute

Mathieu Garand

Sidra Medicine

\section{Basirudeen Kabeer}

Sidra Medicine

Mohamed Alfaki

Sidra Medicine, Doha

\section{Scott Presnell}

Benaroya Research Institute https://orcid.org/0000-0001-9203-6403

\section{Prasong Khaenam}

Benaroya Research Institute

\section{Aaron Ayllon-Benitez}

Bordeaux University https://orcid.org/0000-0002-0684-0364

\section{Fleur Mougin}

Bordeaux University

\section{Patricia Thébault}

Bordeaux University

\section{Laurent chiche}

Hôpital Européen

Noemie Jourde-Chiche

Aix-Marseille University

Theodore Phillips 
Baylor Institute for Immunology Research

\section{Goran Klintmalm}

Baylor Institute for Immunology Research and Baylor Research Institute, Dallas, Texas

\section{Anne O'Garra}

The Francis Crick Institute https://orcid.org/0000-0001-9845-6134

\section{Matthew Berry}

Imperial College Healthcare NHS Trust

\section{Chloe Bloom}

National Institute for Medical Research

\section{Robert Wilkinson}

University of Cape Town, Francis Crick Institute and Imperial College London

\section{Christine Graham}

The Francis Crick Institute

\section{Marc Lipman}

UCL Respiratory, Division of Medicine, Royal Free Campus, University College London

\section{Ganjana Lertmemongkolchai}

Khon Kaen University https://orcid.org/0000-0001-6972-585X

\section{Davide Bedognetti}

Cancer Research Department, Sidra Medicine, Doha

\section{Rodolphe Thiébaut}

Univ. Bordeaux, Department of Public Health, Inserm Bordeaux Population Health Research Centre, Inria SISTM, UMR 1219;CHU de Bordeaux, France; Vaccine Research Institute (VRI), Créteil, Franc https://orcid.org/0000-0002-5235-3962

\section{Farrah Kheradmand}

Baylor College of Medicine

\section{Asuncion Mejias}

The Ohio State University https://orcid.org/0000-0002-5983-8006

\section{Octavio Ramilo}

The Research Institute at Nationwide Children's Hospital https://orcid.org/0000-0002-0565-5239

\section{Karolina Palucka}

The Jackson Laboratory for Genomic Medicine

\section{Virginia Pascual}

Weill Cornell Medicine https://orcid.org/0000-0002-8806-8426

Jacques Banchereau

Jackson Laboratory https://orcid.org/0000-0003-3535-7221

\section{Damien Chaussabel ( $\nabla$ dchaussabel@sidra.org )}

Sidra Medicine, Doha https://orcid.org/0000-0002-6131-7242 
Keywords: human immunology, transcriptome, gene expression, systems biology, network analysis, modular repertoire

Posted Date: September 10th, 2020

DOI: https://doi.org/10.21203/rs.3.rs-58602/v1

License: (c) (1) This work is licensed under a Creative Commons Attribution 4.0 International License. Read Full License

Version of Record: A version of this preprint was published at Nature Communications on July 19th, 2021. See the published version at https://doi.org/10.1038/s41467-021-24584-w. 
1 TITLE: Development of a Fixed Repertoire of Blood Transcriptome Modules Based on Coexpression Patterns Across Immunological States

3 AUTHORS:

4 Matthew C Altman ${ }^{1,2, \#^{*}}$, Darawan Rinchai ${ }^{3 * *}$, Nicole Baldwin ${ }^{4}$, Mohammed Toufiq ${ }^{3}$, Elizabeth

5 Whalen $^{1}$, Mathieu Garand ${ }^{3}$, Basirudeen Ahamed Kabeer ${ }^{3}$, Mohamed Alfaki ${ }^{3}$, Scott Presnell ${ }^{1}$,

6 Prasong Khaenam ${ }^{1}$, Aaron Ayllon Benitez ${ }^{5}$, Fleur Mougin ${ }^{5}$, Patricia Thébault ${ }^{6}$, Laurent Chiche ${ }^{7}$,

7 Noemie Jourde-Chiche ${ }^{8}$, J Theodore Phillips ${ }^{4}$, Goran Klintmalm ${ }^{4}$, Anne O’Garra ${ }^{9,10}$, Matthew

8 Berry $^{11}$, Chloe Bloom ${ }^{10}$, Robert J Wilkinson ${ }^{12,13,14}$, Christine M Graham ${ }^{9}$, Marc Lipman $^{15}$,

9 Ganjana Lertmemongkolchai ${ }^{16}$, Davide Bedognetti ${ }^{3}$, Rodolphe Thiebaut ${ }^{5}$, Farrah Kheradmand $^{17}$,

10 Asuncion Mejias ${ }^{18}$, Octavio Ramilo ${ }^{18}$, Karolina Palucka ${ }^{4,19}$, Virginia Pascual ${ }^{4,20}$, Jacques

11 Banchereau ${ }^{4,19}$, Damien Chaussabel ${ }^{1,3^{*}}$

13 AFFILIATIONS:

141 Systems Immunology, Benaroya Research Institute, Seattle, Washington, USA

152 Division of Allergy and Infectious Diseases, University of Washington, Seattle, Washington,

$16 U S A$

173 Research Branch, Sidra Medicine, Doha, Qatar

184 Baylor Institute for Immunology Research and Baylor Research Institute, Dallas, Texas, USA

195 Inserm U1219 Bordeaux Population Health Research Center, Bordeaux University, Bordeaux,

20 France

216 LaBRI, CNRS UMR5800, Bordeaux University, Bordeaux, France 
227 Department of Internal Medicine, Hospital Europeen, Marseille, France

238 Aix-Marseille University, C2VN, INSERM 1263, INRA 1260, Marseille, France

249 Laboratory of Immunoregulation and Infection, The Francis Crick Institute, London, UK

2510 National Heart and Lung Institute, Imperial College London, London, UK

2611 Royal Cornwall Hospitals NHS Trust, Truro, UK

2712 The Francis Crick Institute, London, UK

2813 Department of Infectious Disease, Imperial College, London, UK

2914 Wellcome Center for Infectious Diseases Research in Africa and Department of Medicine,

30 Institute of Infectious Diseases and Molecular Medicine, University of Cape Town Observatory

31 7925, Republic of South Africa

3215 UCL Respiratory, Division of Medicine, University College London, London, UK

3316 Centre for Research and Development of Medical Diagnostic Laboratories, Faculty of

34 Associated Medical Sciences, Khon Kaen University, Khon Kaen, Thailand

3517 Baylor College of Medicine, Houston, Texas, USA

3618 Abigail Wexner Research Institute at Nationwide Children's Hospital and the Ohio State

37 University School of Medicine, Columbus, Ohio, USA

3819 The Jackson Laboratory for Genomic Medicine, Farmington, Connecticut, USA

3920 Weill Cornell Medicine, New York, New York, USA

40

41 \# Equally contributing authors

42 *To whom correspondence may be addressed: 


\section{CONTACT INFORMATION:}

45 Matthew C Altman, MD, Systems Immunology Division, Benaroya Research Institute, 1201

46 Ninth Avenue, Seattle, WA 98101, USA. Tel. +1 206287 5648, Fax. 206287 5682, E-mail:

47 maltman@,benaroyaresearch.org

48 Darawan Rinchai, Cancer Program, Sidra Medicine, PO Box 26999 Al Luqta Street, Doha,

49 Qatar. Tel. +974 4003 7413, E-mail: drinchai@sidra.org

50 Damien Chaussabel, PhD, Immunology Program, Sidra Medicine, PO Box 26999 Al Luqta

51 Street, Doha, Qatar. Tel.+974 4003 7611, E-mail: dchaussabel@sidra.org

52

53

54

55

56

57

58

59

60 
64 ABSTRACT

65 As the capacity for generating large scale data continues to grow the ability to extract meaningful 66 biological knowledge from it remains a limitation. Here we describe the development of a new 67 fixed repertoire of transcriptional modules. It is meant to serve as a stable reusable framework for 68 the analysis and interpretation of blood transcriptome profiling data. It is supported by customized 69 resources, which include analysis workflows, fingerprint grid plots data visualizations, interactive 70 web applications. These provide access to a vast number of module-specific functional profiling 71 reports, reference transcriptional profiles, and give users the ability to visualize changes in 72 transcript abundance across the modular repertoire at different granularity levels. A use case 73 focusing on a set of six modules comprising interferon-inducible genes is also provided. Taken 74 together, this well-characterized set of modules may be employed for the interpretation and 75 benchmarking of blood transcriptome profiles obtained within and across patient cohorts.

76

77

78

79

80

81

82

83

84 KEYWORDS: human immunology; transcriptome; gene expression; systems biology; network 85 analysis; modular repertoire 
INTRODUCTION:

87 Technology advancements over the past two decades paired with improvements in costeffectiveness have greatly increased the capacity to conduct large-scale molecular profiling in biomedical research. In translational settings, these advances have enabled the measurement of molecular phenotypes at very high resolutions, reaching the point where it has become possible to routinely generate whole genome, proteome, metabolome, microbiome and transcriptome profiles on an almost routine basis (1). The current bottleneck is in interpreting the biological significance or potential clinical implications of these molecular signatures.

Our group has developed co-expression modules and used them as a framework to analyze and interpret blood transcriptome data (2-4). Importantly, we have used the modules as a fixed framework: transcriptional modules are not generated each time a new dataset needs to be analyzed; instead, the same set of pre-existing modules is reused to analyze each new dataset. Consequently, our team has released only two different module repertoires over a 12-year period, which we and others have used to analyze numerous blood transcriptome datasets [e.g. (5-8)]. Other blood transcriptome frameworks have been generated (9), as well as those with a more generic transcriptional module repertoire (10). An inherent benefit of using a collection of gene sets is that it permits the reduction of high-dimensional data. Using a fixed repertoire of modules based on co-expression analyses across a large dataset collection, improves the robustness of 104 analyses of small datasets (10). As such, efforts can rather focus on the functional interpretation of the collection of gene sets constituting the repertoire. Furthermore, using the same fixed

106 framework to analyze multiple independent datasets means that cross-study comparisons and 107 interpretation are easier and more reliable. With the construction of this new repertoire we aimed 
109 limited to 7 in our earlier attempt. Secondly, considerable efforts were deployed to improve 110 resources to support analysis and interpretation.

112 new modular repertoire has been expended considerably compared to the first and second 113 generation repertoires. Specifically, 16 input datasets were employed, comprising 985 unique 114 blood transcriptome profiles from: patients with autoimmune, infectious or inflammatory diseases; 115 cancer patients; liver transplant recipients; and pregnant mothers. We describe here how we 116 developed the analysis workflows for group comparison and individual molecular 117 "fingerprinting". Ad hoc data visualization strategies are presented as well, namely the use of 118 module fingerprint grids and heatmaps to represent the data. We also provide access to extensive 119 functional annotations for each of the 382 modules comprising the repertoire via an interactive 120 web application. Reference blood transcriptome fingerprints are also made available via a web 121 application that enables users to explore the changes observed across diseases/studies and among 122 individuals. A focused effort aiming at the characterization of a set of six interferon modules is 123 presented to illustrate the manner in which such resources may be deployed to help unravel the 124 biology underlying blood transcriptome signatures. 


\section{RESULTS}

133 Constitution of a collection of datasets covering a wide range of immune states.

134 The development of transcriptional module repertoires relies on identifying gene co-expression 135 events using transcriptome profiling data as a starting point. For this new blood transcriptome 136 module repertoire, we used 16 datasets (GEO ID: GSE100150) that encompassed 985 individual 137 whole blood transcriptome profiles. Each dataset corresponds to a different pathological or 138 physiological state (Table 1). These datasets were processed in the same facility and run on 139 Illumina HT12 BeadArrays (details are provided in the method section). Similar to our first two 140 repertoires (Table 2), we included data from patients (adult and pediatric) with: systemic lupus erythematosus (SLE), systemic onset juvenile idiopathic arthritis (SoJIA), liver transplants and receiving maintenance immunosuppressive therapy, metastatic melanoma, and infectious diseases

143 [with an expanded range that now includes infections caused by influenza, respiratory syncytial 144 virus (RSV), human immunodeficiency virus (HIV) infections, Mycobacterium tuberculosis, 145 Staphylococcus aureus, and Burkholderia pseudomallei (the agent of Melioidosis) and sepsis 146 caused by other bacteria (Streptococcus pneumoniae, Salmonella spp, Pseudomonas 147 aeruginosa)]. We also added six new conditions to our framework: inflammatory conditions of the 148 skin (juvenile dermatomyositis), lung [chronic obstructive pulmonary disease (COPD)] and 149 circulation (Kawasaki disease); multiple sclerosis (MS); primary immune (B-cell) deficiency; and 150 pregnancy (serving as a physiological variant). Absent from this repertoire (but represented earlier) 151 are patients with type 1 diabetes or with an Escherichia coli infection. repertoire that will prove useful as a generic framework for blood transcriptome data analyses. 


\section{Implementation of a step-wise approach to blood transcriptional module repertoire}

156 construction.

157 After collating the 16 input datasets, we next followed a stepwise process to construct a module 158 repertoire and identify co-expression networks (Figure 1). We used the module construction 159 algorithm that we have previously implemented (the code is provided in Supplementary File 1). 160 This approach followed four main steps: 1) clustering for each individual dataset; 2) co-clustering, 161 where the number of instances that two genes were included in the same cluster was recorded, with 162 the values ranging from 0 to 16 (i.e. co-clustering observed in none or all of the datasets, 163 164

respectively); 3) constructing a weighted co-expression network, where the edges between the genes represent at least one co-clustering event in one of the input datasets and the weight is assigned based on the total number of co-clustering events; (supplementary Figure 1); and 4) mining the resulting network to identify highly inter-connected sub-networks that form the modules.

Constituting a module repertoire in this manner is thus entirely data-driven: it does not rely on any a priori information about gene interactions or functions. In total, we identified 382 modules comprising 14,168 transcripts $(95.8 \%$ of the transcripts detected in this dataset collection).

\section{Development of module-level analysis workflows and visualizations}

A key characteristic of the gene sets collected via the process described above is that, by construction, changes in abundance of the corresponding transcripts within a given module will tend to be coordinated. As such, it should be possible to use these modules as a "framework" to: 1) identify functional convergences among the genes that comprise each set, and 2) summarize 


\section{Module repertoire construction}
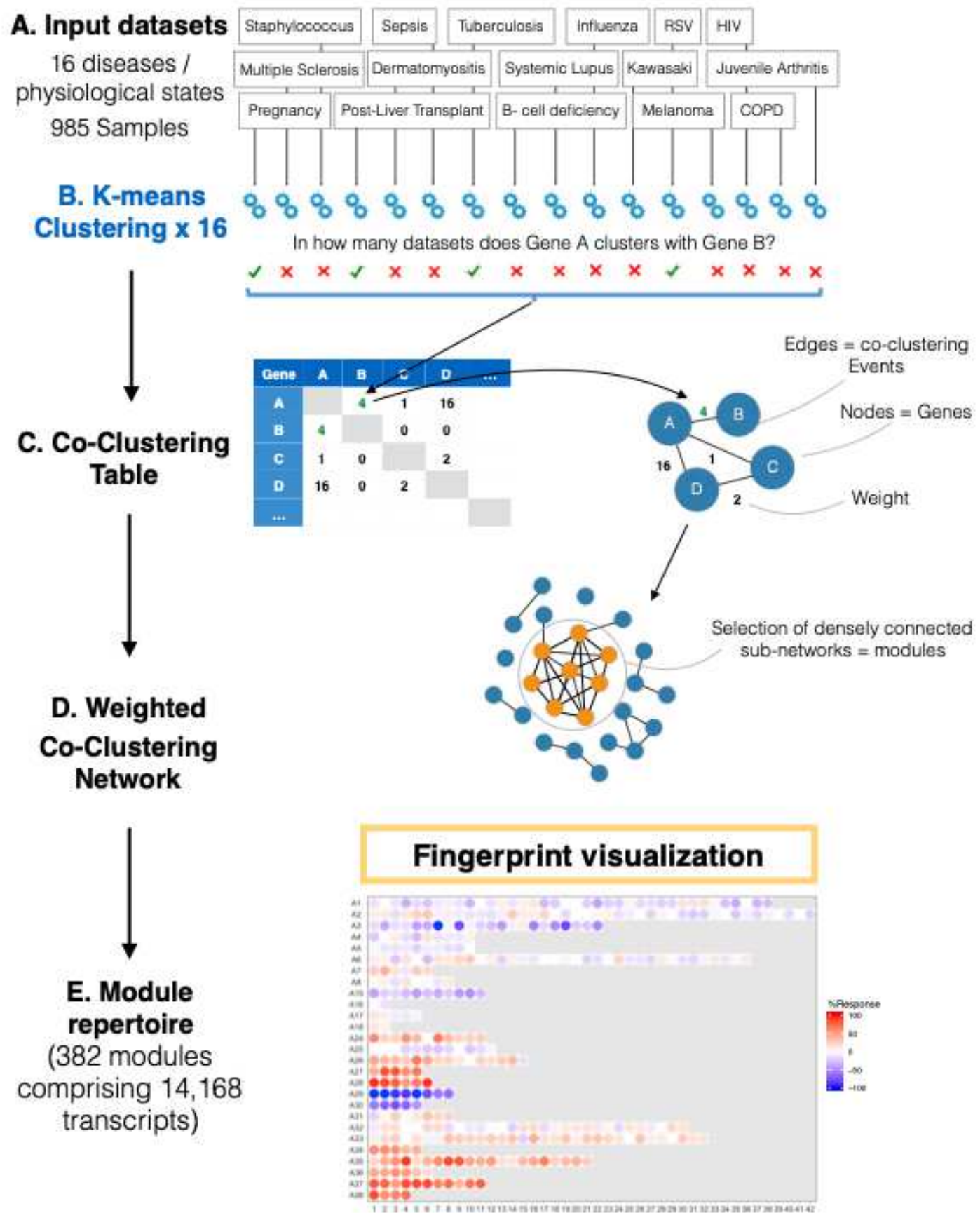

Figure 1: The module repertoire construction process. A. A collection of 16 transcriptome datasets spanning a wide range of immunological and physiological states were used as input. B. Each dataset was independently clustered via k-means clustering. C. Gene co-clustering events were recorded in a table, where the entries indicate the number of datasets in which co-clustering was observed for a given gene pair. D. The co-clustering table served as the input to a weighted co-clustering graph (see also Supplementary Figure 1), where the nodes represent genes and the edges represent co-clustering events. E. The largest, most highly weighted sub-networks among a large network (here constituting 15,132 nodes) were identified mathematically and assigned a module ID. The genes constituting this module were removed from the selection pool and the process was repeated, resulting in the selection of 382 modules constituted by 14,168 transcripts. 
178 changes in overall transcript abundance related to pathological processes or therapeutic 179 interventions.

We determined the gene composition of each of the 382 modules (supplementary file 2),

181 The average number of genes per module as 37.1 , the median as 26.5 and the range as $12-169$.

182 Functional profiling and enrichment results were generated using multiple tools (GSAn, Literature

183 Lab, IPA, DAVID, KEGG, Biocarta, OMIM, and GOTERM). We also determined the extent of 184 overlap with the previous modular repertoire and a set of modules constituted at Emory University 185 and our previous generation of modules (9). For module-level analyses, we determined the proportion of the constitutive transcripts that differ in abundance levels between study groups (e.g. cases vs. controls; pre-treatment vs. post-treatment). By this approach, two values, corresponding to the percent of transcripts that are (i) increased and (ii) decreased, are derived. The cutoff points 189 can be chosen based on user preferences. For example, cutoffs can be based on statistics, fold 190 changes and/or differences with or without multiple testing correction for group comparisons.

From here, the extent of differential expression at the module level can be displayed as a "fingerprint", assigning each module to a fixed position on a grid plot and color-coding it according to the level of increased or decreased abundance for the constituting transcripts (Figure 2). For this we then performed a second tier of clustering to group the 382 modules into 38 "aggregates", 195 with each row on the grid displaying the modules corresponding to one such aggregate. Segregation into distinct aggregates was based on similarities in abundance levels observed across 197 the collection of 16 datasets. By this approach, we derived two levels of granularity (i.e. modulelevel vs. module aggregate-level) with the number of variables for interpretation constrained to a more manageable number. The overall result is that changes in expression levels for each row on 200 the fingerprint grid will tend to be coordinated, which was not the case of prior iterations of such 

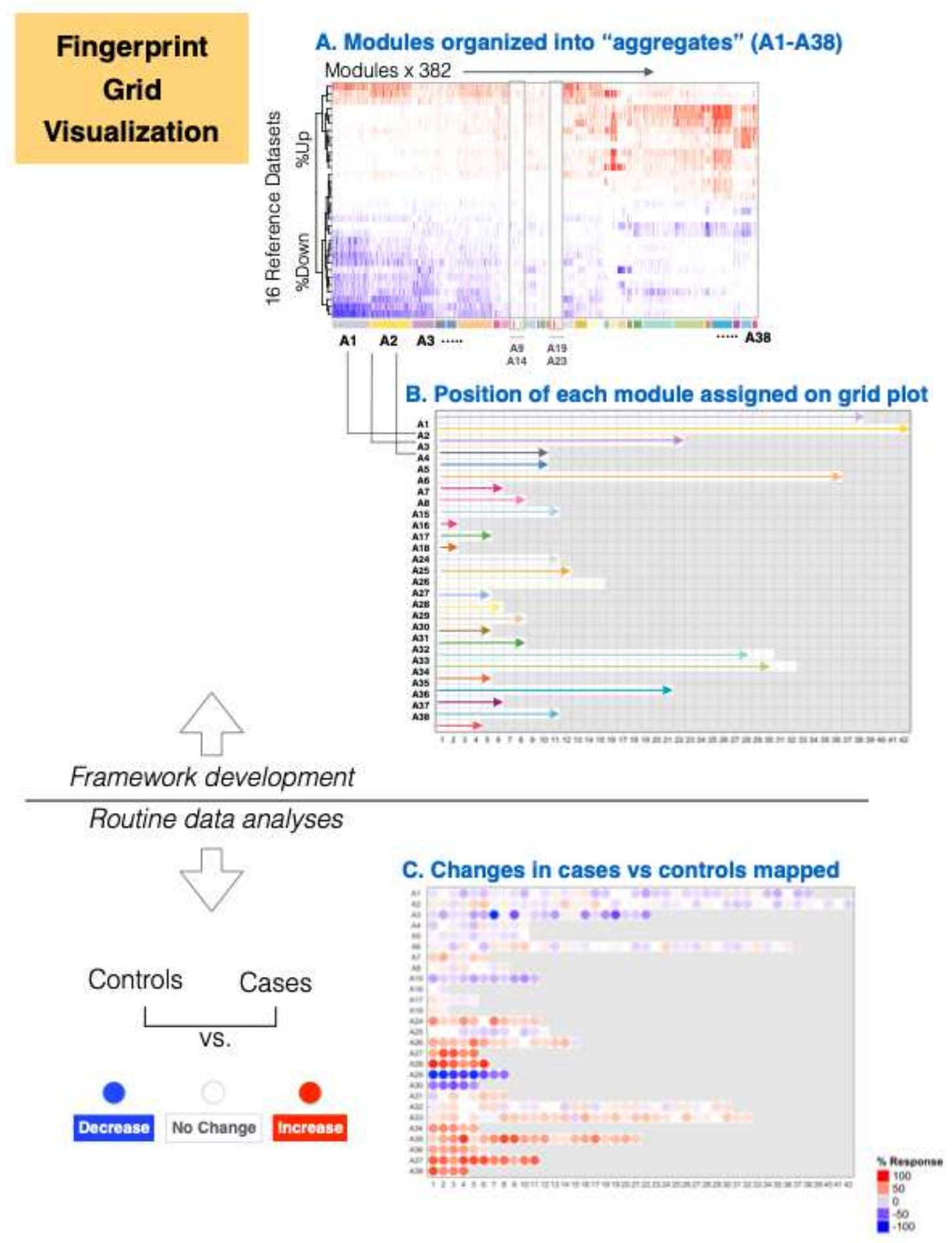

Figure 2: The development of module fingerprint grids. A. The modules were arranged onto the grid as follows: the master set of 382 modules was partitioned into 38 clusters (or aggregates) based on similarities among their module activity profiles across the sixteen reference datasets (A1-A38). B. A subset of 27 aggregates comprising 2 modules or more in turn occupied a line on the grid. The length of each line was adapted to accommodate the number of modules assigned to each cluster. The format of the grid was fixed for all analyses carried out using this modular framework. C. Changes in transcript abundance at the module level were mapped onto this grid and represented by color spots of varying intensity. 
201 fingerprint grids (Figure 3). Some degree of functional convergence can thus be observed within 202 a given row of modules. As an example, in our fingerprint we found that row A1 comprised several 203 modules associated with lymphocytes, while row A28 comprised six distinct "interferon modules" 204 and rows A33 and A35 comprised a number of modules functionally associated with inflammation. the positions of modules on the grid being fixed it becomes for instance possible to rapidly identify the key changes associated with a given pathology or immunological state.

\section{Illustrative use case of fingerprint grid plot representations}

210

We next illustrate the analysis and visualization approach described above with a use case. It will

211 primarily focus on SLE, a disease which blood transcriptome signature has been well-

212 characterized. Fingerprints of other reference disease cohorts employed for module construction

213 will be included to provide additional context.

As mentioned, data interpretation is facilitated by tiered dimension reduction: the first vertical reading of the fingerprint grid permits visualization of changes across the aggregates, while the horizontal reading permits visualization of changes within an aggregate and across modules. As pointed out earlier, in the fingerprint grid, the number and intensity of the spots

218 represents qualitative as well as quantitative differences. As an example, we compared the transcriptome profiles among 55 pediatric patients with SLE and 14 healthy control subjects (Figure 3). We identified an interferon-dominated signature (A28) accompanied by modules associated with cell cycle (A27 and A29, including antibody production). An increase in the

222 abundance of modules associated with inflammation and neutrophils (A35) - a hallmark of the

223 SLE transcriptome signature was also observed. These changes were accompanied by a decrease 
224 in transcript abundance, which was more apparent for some modules belonging to aggregates A1, 225 A2 and A3 which are arrayed across the first three rows of the fingerprint grid. More specifically, 226 for the module aggregate A1, the most marked decreases were observed for modules associated 227 with protein synthesis (dark purple color at positions 1, 5, 11 and 19 on row A1).

It is possible to go a step further and "aggregate" the changes observed by row, thereby 229 reducing the dimensions for a given dataset even further. In this case, we reduced the dataset from 230382 modules to 27 "aggregates" (Figure 3A). Users can decide taking this extra step depends on 231 the desired level of resolution: module-level or aggregate-level. For example, mapping changes at 232 the aggregate level, the simplest framework possible, is optimal when looking at signatures in a 233 broader context. But at the same time our earlier work showed that distinct interferon modules are 234 biologically and clinically meaningful, also it can also be indicated to work instead at the module235 level (11).

We generated fingerprint grid plots for each of the sixteen diseases or physiological states 237 (Supplementary file 3); six module fingerprints are shown in Figure 3 as an illustration. In brief, 238 among these we found that blood transcriptome perturbations were most widespread in patients 239 with MS and patients with a Staphylococcus aureus infection (Figure 3B) with opposing patterns 240 of change. Changes associated with COPD or stage IV melanoma (Figure 3C) were most subtle 241 but nonetheless distinct, with differences in transcript abundance compared to control subjects 242 most visible for aggregates concerning oxidative phosphorylation, monocytes, inflammation 243 (A24-A26), erythrocytes and neutrophil activation (A36-A38). Interferon signatures (A28) were a 244 salient trait in patients with SLE (Figure 3A). We also found differences in the intensities of sets 245 of modules associated with inflammation between these two diseases (A33-A35). 
A. SLE patients $(\mathrm{N}=55)$ / Control subjects $(\mathrm{N}=14)$

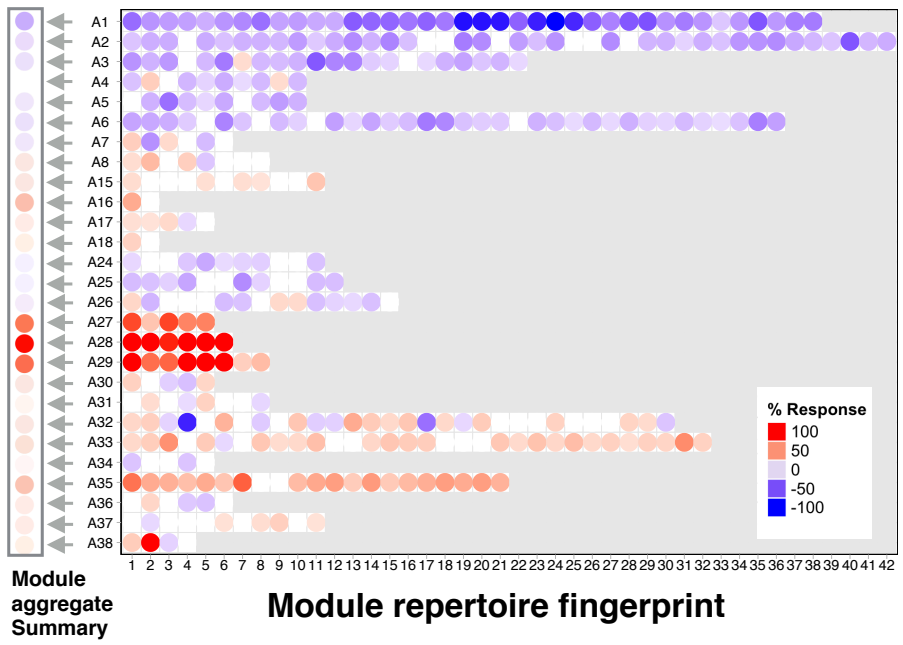

B.

MS

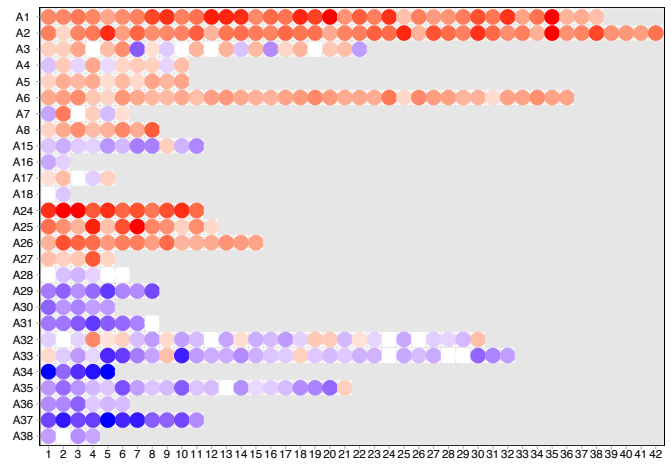

C. COPD

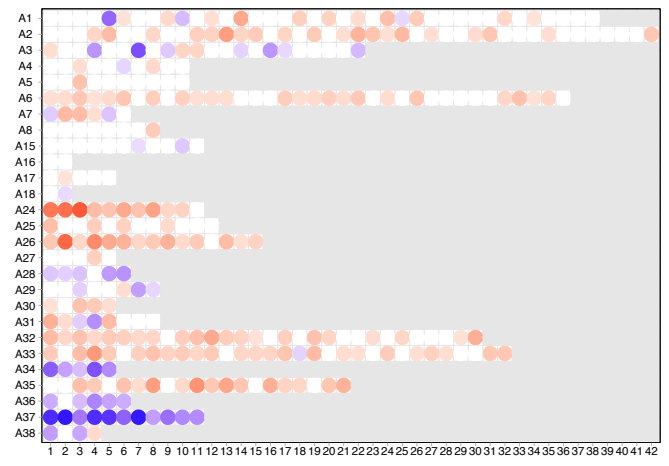

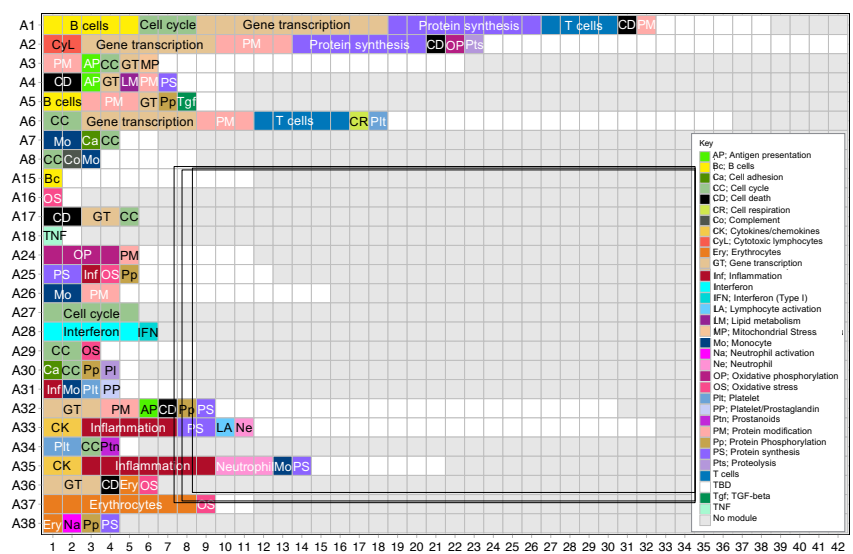

Module annotation grid
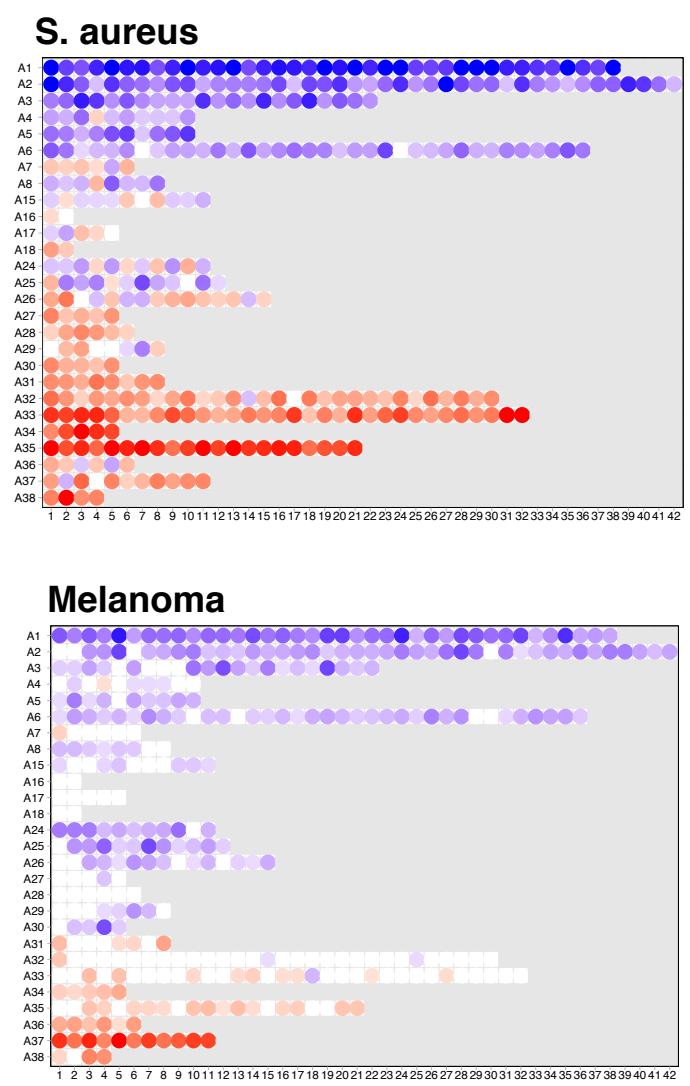

Figure 3: Fingerprint grid plots. A. Prototypical fingerprint grid plot: Changes in blood transcript abundance for patients with SLE compared to healthy controls are represented on a fingerprint grid plot for this illustrative use case. The modules occupy a fixed position on the fingerprint grid plots (see Figure 2). An increase in transcript abundance for a given module is represented by a red spot; a decrease in abundance is represented by a blue spot. Modules arranged on a given row belong to a module aggregate (here denoted as A1 to A38). Changes measured at the "aggregate-level" are represented by spots to the left of the grid next to the denomination for the corresponding aggregate. The colors and intensities of the spots are based on the average across each given row of modules. A module annotation grid is provided where a color key indicates the functional associations attributed to some of the modules on the grid (top right). Positions on the annotation grid occupied by modules for which no consensus annotation was attributed are colored white. Positions on the gird for which no modules have been assigned are colored grey. B-C. Fingerprint grid plots for additional reference datasets. 
Taken together, this use case demonstrates the use of "fingerprint" representations.

247 Notably, fixing positions on the grid permits to overlay functional annotations associated with each modular signature, and the use reference collections of fingerprints for comparative interpretation. However, while this representation can be used as a complement, it altogether does not replace the more traditional heatmap representations.

\section{In depth functional annotation of fixed transcriptional module repertoires}

Based on earlier iterations, the expectation is for this repertoire to be of use over a period spanning at least 5-6 years. We thus put great effort into functionally annotating the repertoire. We followed two main annotation approaches: (1) concurrently running ontologies, pathways or literature-term profiling analyses; and (2) determining for each module the patterns of gene expression for select reference transcriptome datasets. We compiled the resulting information for all 382 modules and have since made it available via an interactive web application Here, it is possible to zoom in and out, determine spatial relationships and interactively browse the very large compendium of analysis reports and heatmaps generated as part of our annotation efforts. (Figure 4; Links for each aggregate are listed in Table 3.; A demonstration video is available: https://youtu.be/8ajESET2mqI) Below, we describe briefly how we conducted our annotations 263 (see also Experimental Procedures). using DAVID (12), GOTERM, and GSAn (13). GSAn interactive reports were uploaded to a custom web portal (https://ayllonbe.github.io/modulesV3/index.html). We also performed

267 pathway enrichment analyses using KEGG, Biocarta and Ingenuity Pathway, as well as literature 


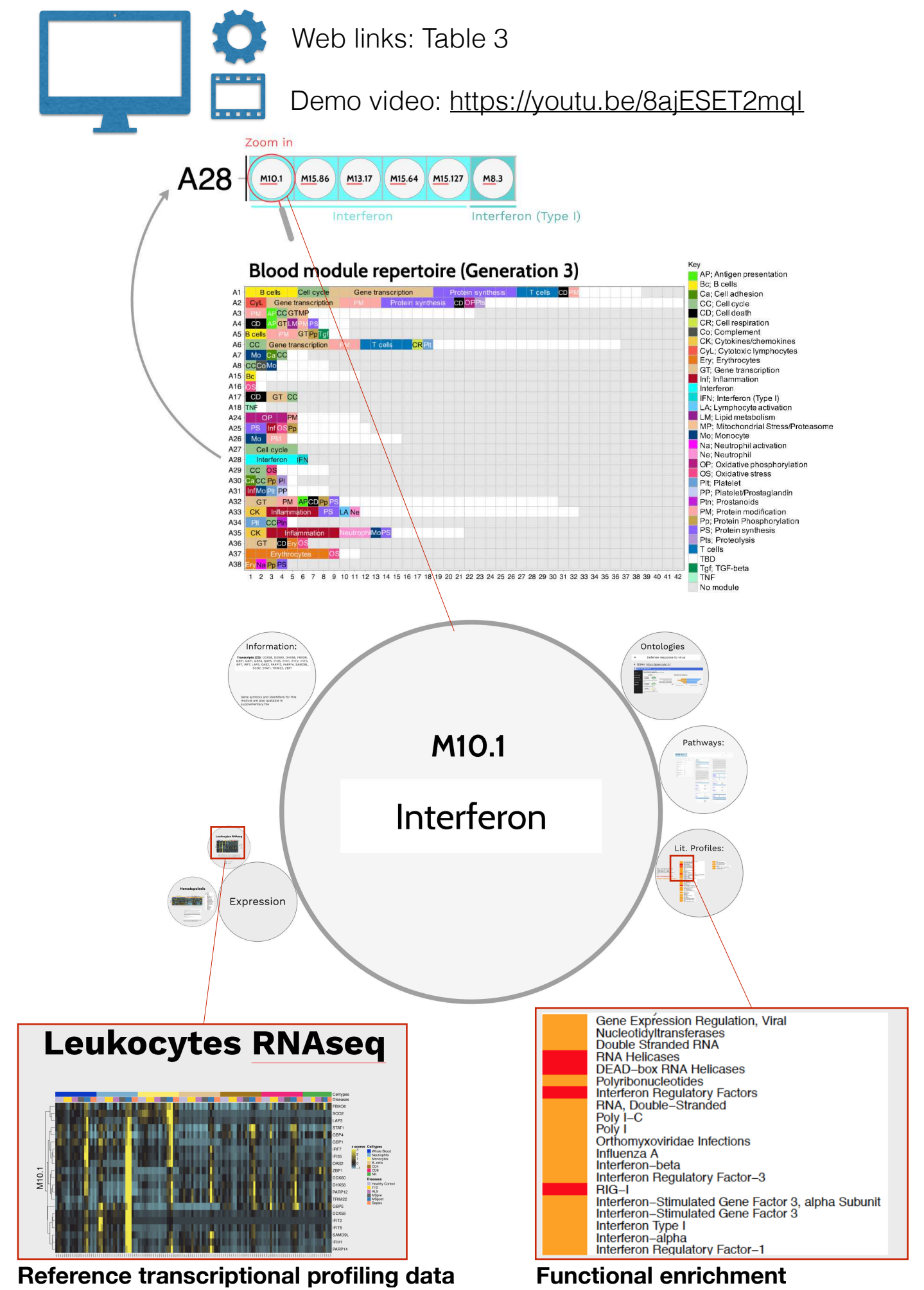

Figure 4: Functional annotation of the transcriptional module repertoire. An interactive application is available to explore the 382 modules comprising the blood transcriptome repertoire. A gene list, along with the ontology, pathway, literature term enrichment and transcriptional profiling data for reference transcriptome datasets (circulating leukocyte populations, hematopoiesis) is provided for each module. Zoom in and out functionalities for close-up examinations of the text and figures embedded in the presentation is possible. Web links providing access to modules within a given aggregate are listed in Table 3. For a demonstration video, please visit: https://youtu.be/8ajESET2mqI. 
269 File 2) and used them to identify convergences and attribute functional titles to the different 270 modules. Functional titles could not be attributed in all cases, due to a lack of convergence or poor 271 enrichment in one or several of the analyses.

272 Step 2 - Expression patterns in reference transcriptome datasets: We used transcriptome datasets 273 as a reference to improve characterization and biological interpretation of the module framework.

274 Two different datasets were used. The first was contributed by Novershtern et al. and comprised 275 the transcriptome profiles of 38 human hematopoietic cell populations (14). The second was 276 contributed by Speake et al. and comprised the RNAseq profiles of six circulating leukocyte 277 populations from patients with various immune-associated diseases (15). We generated heatmaps 278 for each module to display the abundance patterns of the constitutive transcripts for each dataset. necessary for interpreting transcriptome fingerprints generated via module-level analyses. Second, 281 it helps us to improve the attribution of functional titles and roles to the different modules and aggregates. Indeed, although the transcriptional module repertoire is fixed over time, we anticipate that the functional annotations will continue to evolve over its lifespan.

\section{Measuring inter-individual variability for the molecular stratification of patient cohorts}

The analysis and visualization steps presented so far focused on characterizing differences between groups of subjects (e.g. cases and controls). However, it is also important to characterize heterogeneity among groups of patients since inter-individual variability can serve as a basis for the definition of molecular phenotypes and patient stratification. 
Within each module, and for each individual subject, we used fixed cutoffs to count the number of transcripts that increase, decrease or do not change in abundance compared to a baseline 292 value (e.g. absolute fold change in expression and absolute difference in expression vs. average of 293 control samples). The percentage of differentially expressed genes for each module are then 294 computed. These percentages are equivalent to values derived from group comparisons, except 295 that they are derived for each individual sample.

The sepsis cohort comprised in the reference dataset collection was used to illustrate how 297 this approach can be employed for assessment of inter-individual variability for a given pathology 298 (Figure 5). Changes in transcript abundance across was found to be highly consistent across 299 patients for some module aggregates. This was for instance the case of aggregate A1 (broadly 300 associated with lymphocytic cells/responses), with consistent decreases in transcript abundance 301 observed across patients. Conversely, consistent increases were observed for modules comprising 302 aggregate A35 (broadly associated with inflammatory neutrophil responses). In this case 303 differences were observed in the intensity of the response. Other module aggregates, however, 304 showed more mixed responses, which was the case of A37 (erythroid cells), A33 (functional 305 association to be defined) or A28 (interferon response).

A web application was developed as a resource to explore the inter-individual differences 307 for a given disease, module aggregates or a combination of aggregates 308 (https://drinchai.shinyapps.io/dc_gen3_module_analysis/\#; video: https://youtu.be/y_7xKJo5e4 309 ). This application permits the generation of fingerprint grid plots and heatmaps representing 310 module aggregates activity across the 16 reference datasets (Figure 6). 


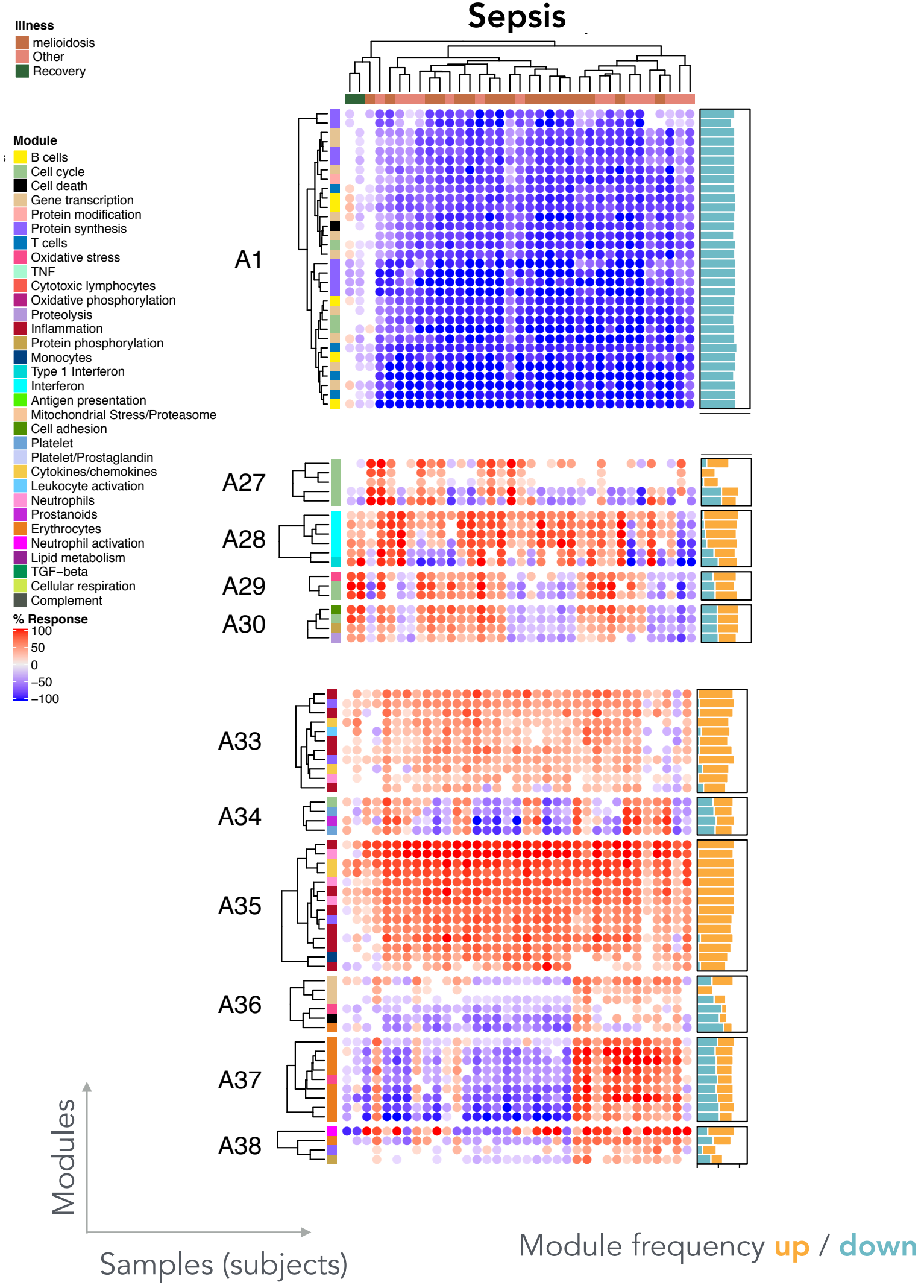

Figure 5: Individual-level module heatmap. Changes in transcript abundance were determined at the individual level across all modules constituting the repertoire. These changes are represented on a heatmap, where an increase in abundance of a given module is represented in red, and a decrease in abundance is represented in blue. The subjects are organized as columns and the modules as rows. The ordering on the heat map was determined by hierarchical clustering. 


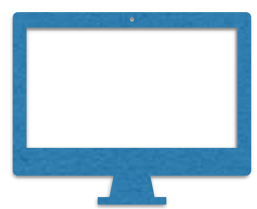

S. $h$ https://drinchai.shinyapps.io/dc_gen3_module_analysis/\# $+\cdots$

Demo video: https://youtu.be/y__7xKJo5e4

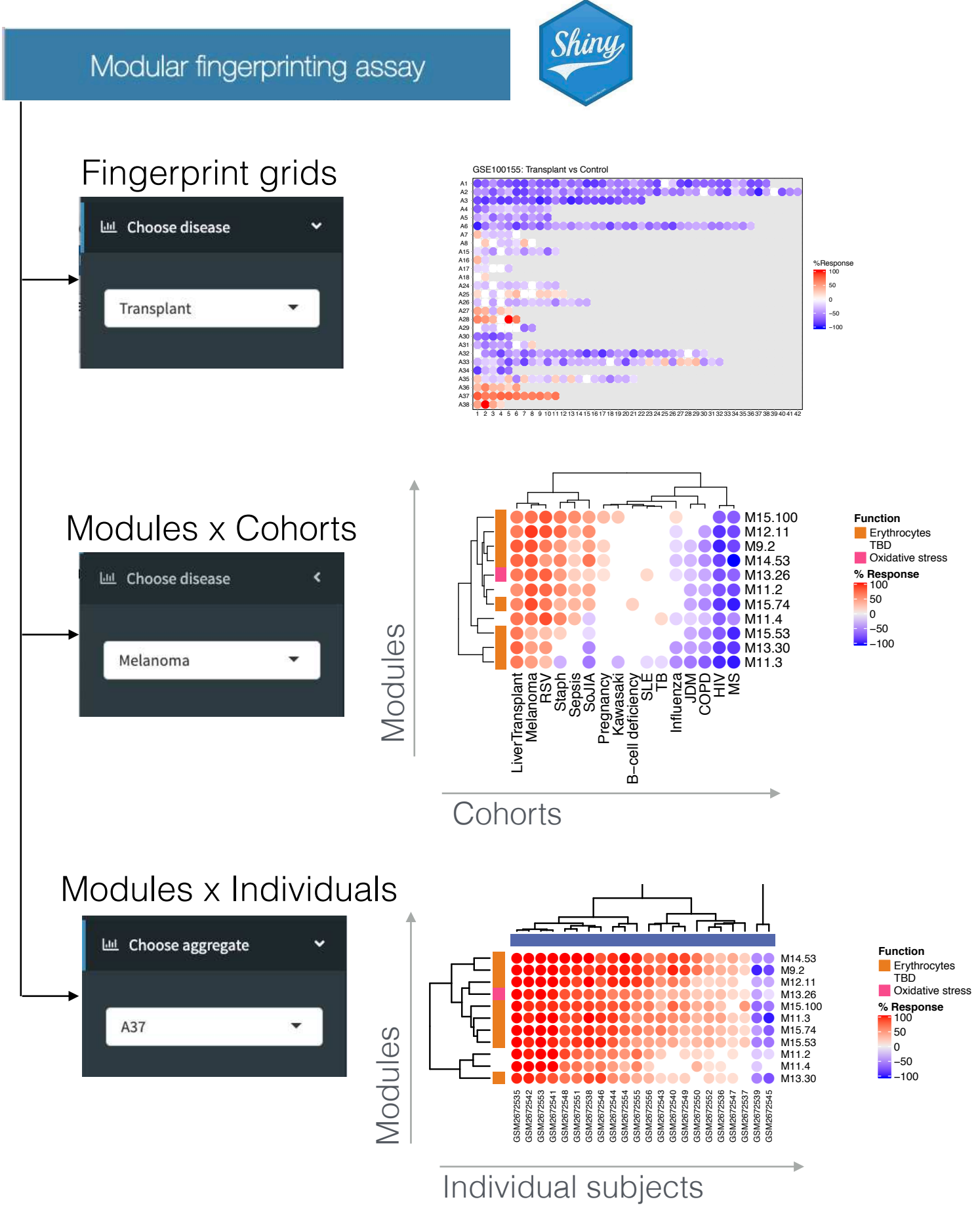

Figure 6: Web application to visualize multi-tiered module fingerprinting. An application was developed to explore the changes in transcript abundance at the module level across the 16 reference datasets used to construct the repertoire. Three types of plot can be displayed and exported: 1) fingerprint grids; 2) module heatmaps displaying changes in abundance in modules comprising a given aggregate across the 16 reference datasets; and 3) module heatmaps displaying changes in abundance in modules comprising a given aggregate across individuals constituting a given dataset. The application is accessible via this link: https://drinchai.shinyapps.io/dc gen3 module analysis/\#. Demonstration video: https://youtu.be/y 7xKJo5e4. 
Altogether, as illustrated by the sepsis use case provided here, deriving individual modular

312 fingerprints can provide a means to achieve molecular stratification of patient cohorts. However,

313 the biological and clinical relevance of such distinct molecular phenotypes would still remain to

314 be determined in follow on analysis steps.

315 Profiling the abundance of A28 interferon-inducible genes at the aggregate-level across 316 reference patient cohorts

317 The earlier sections dealt first with the approach implemented for the construction and 318 characterization of the fixed transcriptional module repertoire. Analysis and visualization 319 strategies for both group-level and individual-level comparisons were presented next. Now we 320 would like to go over a use case focusing on the dissection of changes in abundance for module 321 aggregate A28 (interferon responses). We first start here from the highest possible perspective, 322 examining changes in abundance for the A28 aggregate across reference disease cohorts.

A heatmap was derived showing patterns of abundance of a subset of 27 module aggregates comprising two or more modules across 16 health states (Figure 7A). The first order of separation 325 grouped acute HIV infection, MS, juvenile dermatomyositis and COPD in one cluster. The remaining 14 states grouped into a second cluster, with RSV infection presenting as an outlier. The main trend driving the dichotomy between the first four diseases and the rest was an overall 328 suppression of modules associated with inflammation and/or myeloid cell responses (A34-A38), 329 accompanied by an increase in modules corresponding to aggregates associated, in part, with 330 lymphocytic responses (A1-A8). The factors underlying these two distinct, "overarching" 331 signatures are unclear; diseases belonging to either group can exhibit marked interferon signatures 332 (e.g. acute HIV infection in one cluster, and SLE or influenza infection in the other cluster). The 333 circle plots shown on this figure illustrate at a more granular module and gene-level the changes 
334 that are being represented by spots on this heatmap. The gene composition for each of the six module is shown in one of those circle plots (Figure 7B). The other represent which of the genes among those modules are changed when comparing patients with infection to their respective

337 control groups (Figure 7C). Finally, we added for reference circle plots showing patterns of in 338 vivo response of $\mathrm{A} 28$ genes to administration of IFN $\alpha$ to patients with hepatitis $\mathrm{C}$ infection or of 339 IFN $\beta$ to patients with multiple sclerosis [transcriptome profiling data were made publicly available by the authors $(16,17)]$ (Figure 7D). The plots on this figure show that changes observed at the

341 aggregate level are not always distributed evenly across the six modules constituting aggregate 342 A28, and in turn of genes constituting each of the modules. The response to type I interferons was 343 dominated by a disproportionate increase in abundance of transcripts constituting M8.3 and M10.1. 344 Transcripts forming M15.86, that showed very little changes in response to those treatments, were 345 on the other hand markedly increased during acute HIV and influenza infection (Figure 7C). It is 346 thus possible that this gene set be more specifically induced by interferon gamma. Interferon 347 responses were weaker among RSV patients compared to these two other viral illnesses. Similarly, 348 in the context of bacterial infection the interferon response was most marked in response to TB 349 infection, which is consistent with the literature (8). A large proportion of adult patients comprising 350 the sepsis cohorts were infected with Burkholderia pseudomallei, the intracellular bacteria 351 responsible for melioidosis that also tends to induce higher interferon responses (8). Changes in 352 abundance for transcripts constituting A28 in the context of autoimmune or inflammatory diseases 353 is shown in Supplementary Figure 2.

The visualization schemes employed here already affords some new perspectives about the 355 contribution of different modules and genes to the overall aggregate-level interferon responses. 

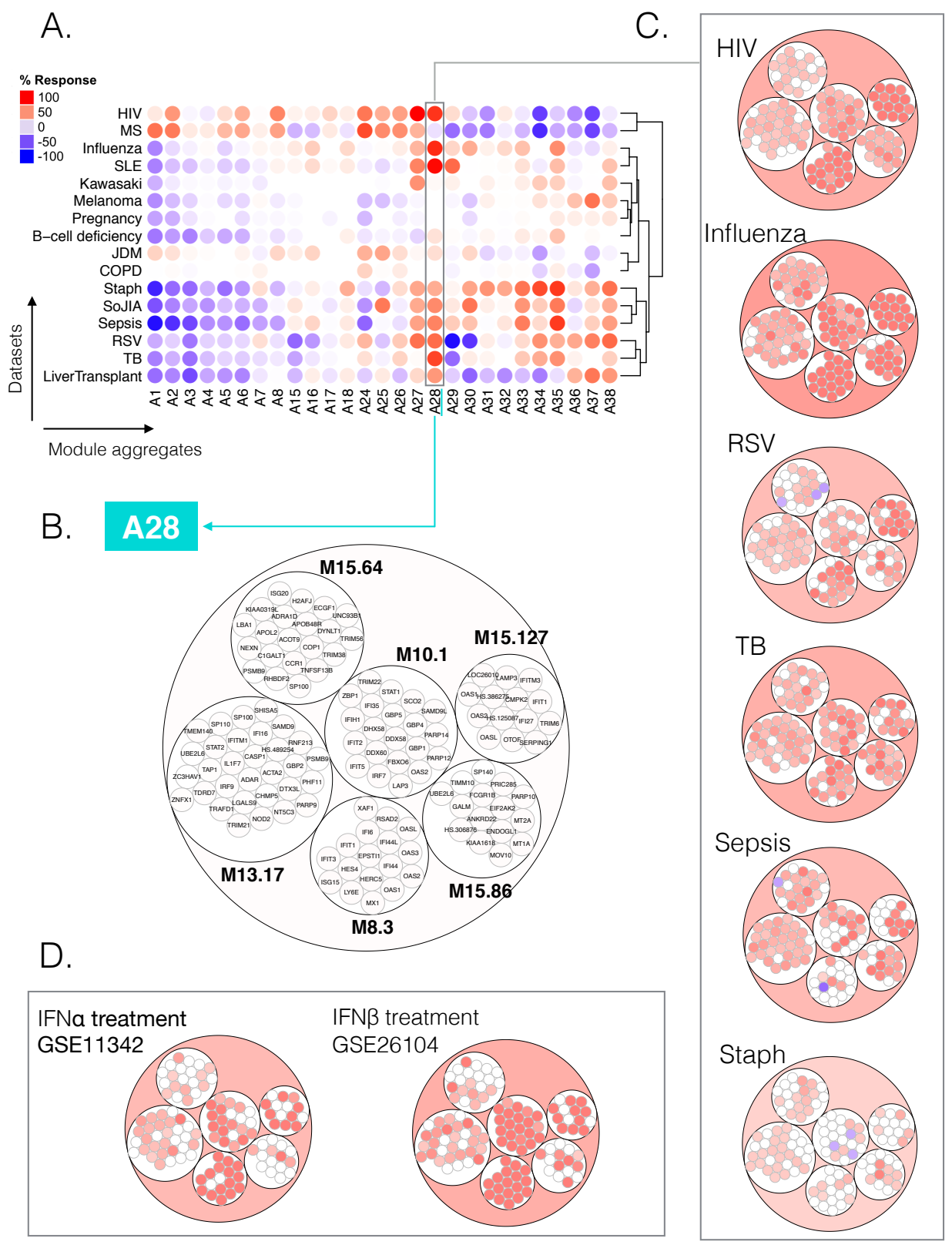

Figure 7: Module aggregate abundance patterns across the 16 disease or physiological states. A. Patterns of changes in transcript abundance at the aggregate and cohort levels. Each column on the heat map corresponds to a "module aggregate", numbered A1 to A38. Modules A9-A14 and A19A24 were excluded as they each only included one module. Each row on the heatmap corresponds to one of the 16 datasets used to construct the module framework. A red spot on the heatmap indicates an increase in abundance of transcripts comprising a given module cluster for a given disease or physiologic state. A blue spot indicates a decrease in abundance of transcripts. No color indicates no change. Disease or physiological states were arranged based on the level of similarity in the patterns of aggregate activity, determined via hierarchical clustering. B. Representation of the modules and genes constituting aggregate A28. The circle plot represents the six modules constituting aggregate 28 , and the transcripts constituting each of the modules. Some genes on the Illumina BeadArrays can map to multiple probes, which explains the few instances where the same gene can be found in different modules. C. Patterns of changes in transcript abundance at the module-level and gene-level for aggregate A28. The circle plots illustrate the changes at the gene-level for this aggregate for $6 / 16$ datasets. The position of the genes on each of these plots is the same as shown in panel B. Genes for which transcript abundance is changed are shown in red (increase) or in blue (decrease). D. Patterns of changes in transcript abundance at the module and gene level for aggregate A28 in subjects treated with IFN $\alpha$ or IFN $\beta$ treatment The circle plots show changes in abundance for A28 transcripts in patients with hepatitis C infection treated with IFN $\alpha$ [GSE11342 (16)] or patients with multiple sclerosis treated with IFN $\beta$ [GSE26104 (17)]. 
356 However other variation in the selection of variables and granularity levels are possible and will

357 be explored next.

Profiling the abundance of A28 interferon-inducible genes at the module-level across reference patient cohorts.

Another perspective was gained next by plotting changes of A28 genes across the same reference cohorts but at the more granular module-level, rather than at the aggregate level. It was also at this level that we chose to compare literature keyword enrichment profiles for each of the A28 364 module.

Functional enrichment analyses showed that all six modules in this aggregate were associated with the interferon response (see https://prezi.com/view/E34MhxE5uKoZLWZ3KXjG/

367 ). The heatmap (Figure 8A) showing literature enrichment profiles highlighted keywords associated with viral pathogens ("hepatitis", "herpes" or "influenza”), as well as host-derived and 369 pathogen-derived molecules ("RIG-I", "Interferon", "Interferon, “double stranded RNA"). From 370 the six modules, four seemed to be "core" interferon modules (M15.86, M10.1, M8.3, M15.127), 371 while the remaining two (M13.17, M15.64) were associated to the interferon literature to a lesser 372 degree. These latter two modules were more strongly associated with the Herpes simplex virus 373 than the other four modules while the four core modules were preferentially associated with 374 hepatitis. The heatmaps available via the Prezi interface, which depict the gene expression profiles 375 for the A28 modules provide additional perspectives. For example, the dataset from Speake et al. 376 comprised samples of patients with MS collected immediately before and $24 \mathrm{~h}$ after the 377 administration of their first dose of interferon- $\beta$ (Supplementary Figure 3 [GEO ID GSE60424); 
378 (15)]. Despite the small number of subjects in this category, we observed a clear pattern of response 379 to interferon in vivo across all six modules. This observation confirms the functional associations 380 obtained from enrichment analyses.

Next, we examined the degree of changes for the six interferon modules across the 16 input 382 datasets (Figure 8B). The first cluster showing the highest induction levels comprised SLE, influenza infection, HIV infection and active M. tuberculosis infection (noted as C1 on the figure). Interferon has antiviral properties; therefore, it is no surprise to see viral infections included in this set. Blood transcriptome profiling studies conducted nearly 20 years ago identified interferon responses in SLE pathogenesis $(18,19)$. More recent profiling also revealed the prominence of this signature in patients with tuberculosis, which contrasts with findings made in other bacterial infections (6). The second cluster (noted as C2) comprised diseases with an "intermediate" level of interferon responses, including RSV infection, sepsis caused by Staphylococcus aureus in pediatric patients and a range of bacterial pathogens in adults, SoJIA and liver transplant recipients receiving maintenance immunosuppressive therapy. The illness conferred by RSV infection has a clinical presentation very similar to that of influenza in pediatric patients. However, studies suggest that interferon responses may be partially inhibited by RSV $(20,21)$.

The final, third cluster was formed by pathologic and physiological states where an 395 increase in abundance of interferon-inducible genes was either modest or inexistent. This cluster included patients with juvenile dermatomyositis and B-cell deficiency (low levels), Kawasaki 397 disease, melanoma, COPD, pregnancy (no increase), and individuals with MS (apparent decrease). 398 The latter observation presents some interest since one line of treatment for multiple sclerosis is 399 consists in administering interferon beta. This should as a result compensate the defect observed 400 here in treatment-naïve individuals. 


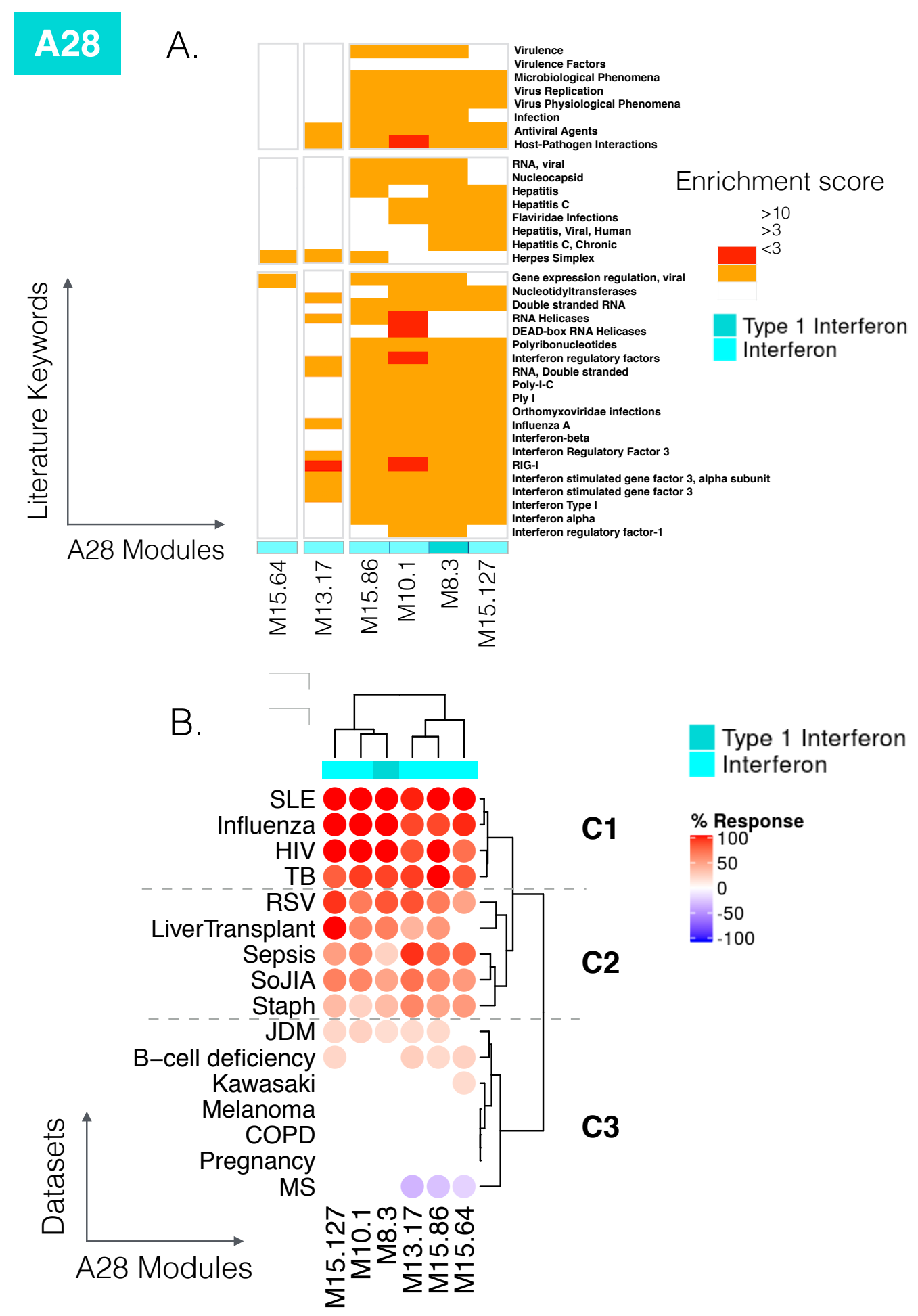

Figure 8: Literature profiles and patterns of changes in abundance across reference datasets for the modules comprising aggregate A28. A. Functional annotation by literature profiling: A portion of a heatmap comprising 382 modules organized as columns, and literature terms organized as rows. The six modules shown are associated with the consensus annotation "Interferon" or "Type 1 Interferon". The clusters of keywords associated with those modules are consistent with this annotation and provide added granularity to the module repertoire for functional profiling and interpretation. B. Changes in abundance across 16 reference datasets: the heatmap (top) represents the changes in abundance of transcripts constituting the six modules comprising aggregate A28 (columns). The modules are functionally associated with interferon responses. The 16 reference datasets are arranged as rows corresponding to different health states. The columns and rows were arranged by hierarchical clustering. Such heatmaps can be accessed and exported for all 16 datasets and 38 module aggregates using the web application: https://drinchai.shinyapps.io/dc gen3 module analysis/\# (under the "MODULES x STUDIES" tab). 
Taken together, this closer examination of annotations for A28 modules and patterns of 402 changes in abundance in different reference datasets provided a clearer picture of its biological 403 significance.

\section{Profiling the abundance of A28 interferon-inducible genes at the module-level across} individual subjects.

407

408

409

410

As described earlier analysis workflows have been developed to determine changes in transcript abundance at the level of individual subjects. This approach, which offers an even more granular perspective, was applied next for molecular stratification of patient cohorts using the six A28 interferon modules.

We show, for instance, that in cohorts where no changes are detectable via comparisons at the overall group level, a minority of patients in fact present the signature. This scenario indeed applies for the cohort of melanoma patients with 3 out of 22 subjects showing some degree of increase in abundance for the six A28 modules, while the majority showed little changes or decrease in abundance. At least four of the patients showed a marked decrease in abundance (Figure 9A). The observation carries potential biological and clinical significance, as interferon activity in patients with melanoma has been previously associated with disease outcomes $(22,23)$. Pathologies with an intermediate A28 signature might induce responses in a somewhat higher proportion of subjects. This is the case of JDM patient cohort that comprises a cluster of 11 patients our of 40 presenting with modular interferon signatures (Figure 9A). Of the 793 articles in PubMed mentioning "Juvenile Dermatomyositis" in their titles, 8 also mentioned "interferon", which indicates that a role for interferon in this disease while not widely acknowledged has 
nonetheless been described (24). In diseases where the role for interferon is well described, such as influenza infection or SLE, increase in A28 modules is widespread. But it is nevertheless possible to find at least a few subjects in each cohort for whom an interferon signature is not present (Figure 9A). Notably in the case of SLE the proportion of interferon negative subjects tends to be higher in adult patient cohorts in comparison to pediatric patient cohorts as the one that is being used for illustrative purposes here. Stratification of an adult SLE cohort based on patterns of abundance of A28 modules is presented next.

We previously showed using our second generation repertoire that the interferon signature characterizing SLE comprises distinct "sub-signatures" at the module level (11). In this earlier work we observed a sequential increase for a set of three interferon modules (including M1.2, M3.4 \& M5.12). M1.2 showed a higher degree of sensitivity, followed by M3.4 and then M5.12. Based on this finding, we could stratify SLE patients based on whether one, two or all three of those interferon modules were activated. By combining functional profiling with a reference dataset, we concluded that the modules responded differently to each interferon type: interferon- $\alpha$ induced an increase in the abundance of genes belonging to M1.2; interferon- $\beta$ induced an increase in the abundance of genes belonging to M1.2 and M3.4; and interferon- $\gamma$ induced an increase in the abundance of genes belonging to M5.12. Next, we sought to determine the equivalence between these three, second generation interferon modules and the six new, third generation interferon modules regrouped in aggregate A28. Based on gene composition, M8.3 and M15.127 mapped to M1.2 (inducible by both interferon- $\alpha$ and $\beta$ ), M10.1 and M15.86 mapped to M3.4 (inducible by interferon- $\beta$ ), and M13.17 and M15.64 mapped to M5.12 (inducible by interferon$\gamma)$. Notably, the latter two modules did indeed segregate from the other four on literature the enrichment profiling heatmap presented earlier (Figure 8A). We also used the six interferon 
A. Melanoma
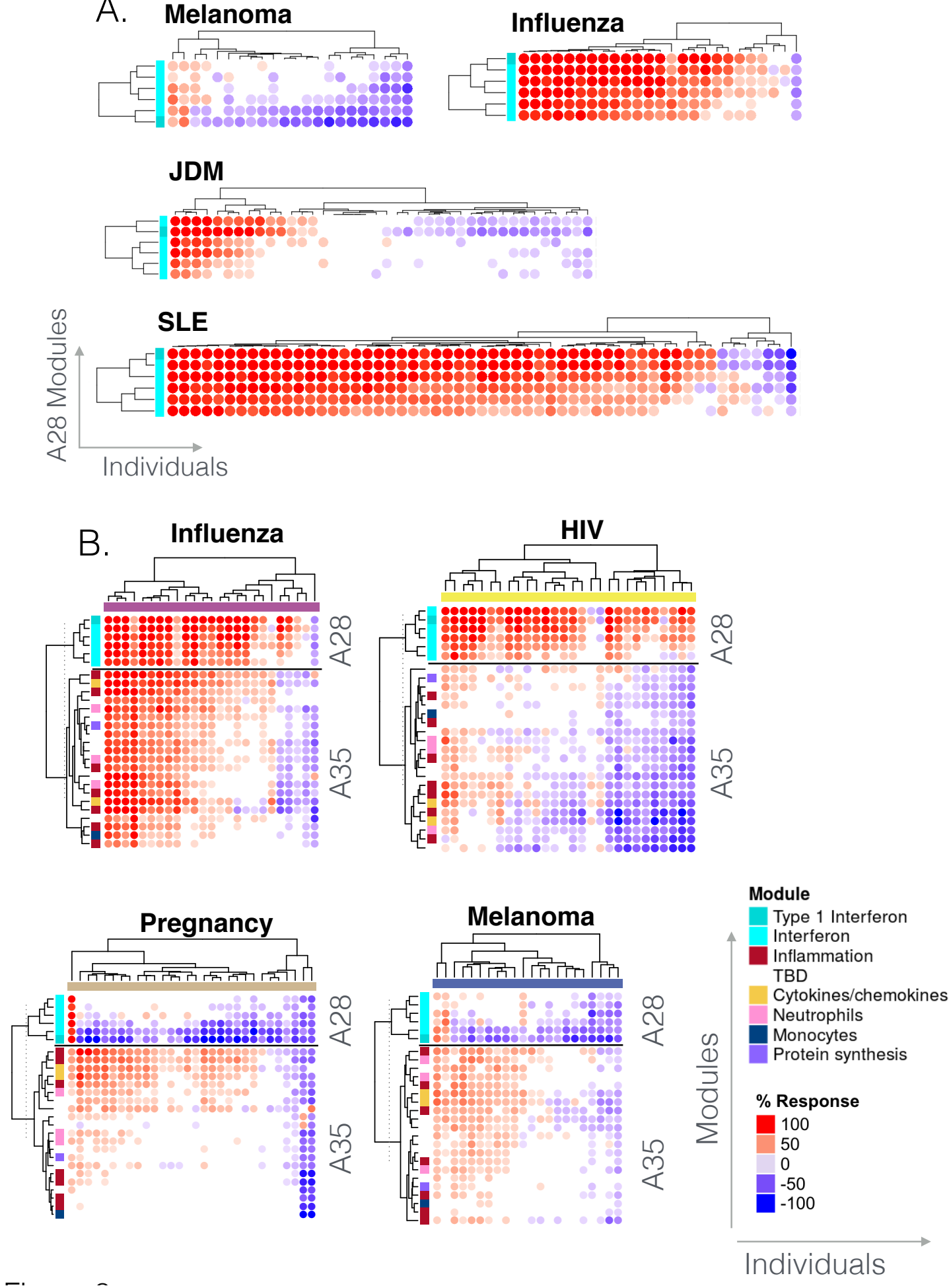

Individuals

Figure 9: Abundance patterns across individuals. A. Changes in abundance for A28 modules. The heatmaps display the changes in abundance for the same six modules (rows) across individuals (columns) in four reference cohorts. The rows and columns on the heatmap are arranged based on similarities in abundance patterns. B. Changes in abundance for A28 and A35 modules. The heatmaps display the changes in abundance of six modules constituting aggregate A28 and 21 modules constituting aggregate A35 (rows) across individuals (columns) in four reference datasets. Functional annotations associated with different modules is indicated by a color code and corresponding legend. Such heatmaps can be accessed and exported for all 16 datasets and 38 module aggregates using the web application: https://drinchai.shinyapps.io/dc_gen3_module_analysis/\# (under the "MODULES x INDIVIDUALS" tab). 
modules to re-classify the adult SLE dataset profiled in our earlier study [GEO ID GSE49454 (11)]. The resulting clustering and stratification mirrored our earlier findings made using the three interferon modules (Supplementary Figure 4). Overall these observations confirm that interferon "sub-signatures" may be employed for patient stratification. This may be relevant in the tailoring of biologics targeting interferon which are under development for the treatment of SLE. However, whether utilizing six modules from the new repertoire would necessarily be an improvement over the three from the second-generation repertoire that were employed previously remains to be determined.

Finally, we wanted to illustrate how further insights could be obtained by examining changes in A28 modules abundance at the individual level concurrently with that of other module aggregates. As an example, we present the changes for both A28 (interferon) and A35 (inflammation) modules (Figure 8B). By combining the two modular signatures, we could assess their relative contributions in a given cohort and identify distinct phenotypes: i.e. interferon or inflammation "positive", "double positive" or "double negative". We found the most contrasting patterns of change in transcript abundance in the sepsis and melanoma cohorts. Here, the abundance of interferon (A28) and inflammation (A35) modules almost uniformly increased in patients with sepsis. By contrast, we only observed increases in these modules in a minority of melanoma patients, with increases of inflammation modules being more widespread than that of interferon modules ( $\sim 50 \%$ compared to $\sim 10 \%$ of subjects, respectively). This finding was paralleled in pregnant women, who represent another immunosuppressive state, although increases in abundance of a subset of A35 modules appeared to be more prevalent in this group than in melanoma patients. Influenza infection was more similar to sepsis but with higher levels of interferon induction and a lower level of inflammation. 
Taken together, the use case presented here illustrates the preliminary stepwise dissection

470 of a given module aggregate and investigation of the underlying biological relevance. Such a

471 process would be to be repeated for other module aggregates. While this is beyond the scope of

472 the present work it is a process that the scalable annotation infrastructure that has been developed

473 here will support. We indeed expect the range of reference datasets and functional profiling 474 approaches available for interpretation to continue to expand. This work should also help 475 determine to what extent subdivision of signatures in distinct modules is warranted.

477 DISCUSSION:

478 We have developed a new resource for the analysis and interpretation of blood transcriptome data.

479 At its core is a fixed, transcriptional module framework. This framework has been constructed via 480 co-expression analyses carried out across a wide range of diseases and physiological states. It 481 constitutes the third iteration released by our group since 2008. Another blood transcriptome 482 repertoire was developed and made available by our collaborators from Emory University [Li et 483 al.: (9)]. While methodological differences exist for the construction of such frameworks the 484 principle remains the same: i.e. utilization of reference datasets for constitution of reusable sets of 485 transcriptional modules.

Collections of gene sets are commonly used for the interpretation of transcriptome profiling 487 data [Gene Set Enrichment Analyses (GSEA): (25) (26)]. Such reference collections are very large, 488 typically numbering tens of thousands of signatures. While they prove useful as a referential for 489 functional interpretation, the majority of the sets are not derived via co-expression analysis. They 490 would therefore not be sufficiently coherent to serve as a basis for dimension reduction, nor were 491 they meant to be used in this fashion. Conversely, the construction of the set of transcriptional 
modules that is presented here is entirely driven by co-expression, and is suitable for reducing dimensions, but is intended for a much narrower use (i.e. the analysis and interpretation of blood transcriptome data). Zhou and Altman recently described the development of another fixed transcriptional module repertoire via co-expression analysis and discuss the advantages of such approaches (27). In brief, they assembled a collection of 2753 public datasets that were deposited in the NCBI GEO that encompassed 97,049 unique transcriptome profiles. They used independent component analysis for resolving co-expression relationships which led to the identification of a set of 139 transcriptional modules. They went on to demonstrate the biological relevance of these sets and advantages their use presents for improving robustness of analyses, especially for smaller datasets. Altogether this article provides additional arguments in favor of the reuse of fixed transcriptional module repertoires, an approach that to date has not seen widespread use so far. The authors chose a very wide breadth of datasets as input and the resulting framework may prove rather generic since transcriptional regulation is largely cell and tissue-specific.

A key characteristic of fixed transcriptional module frameworks is that they are intended for reuse, often over long periods of time. One of the major implications is that greater amounts of effort can be dedicated to the annotation and development of $a d$ hoc visualization tools. This is obviously not possible when new module repertoires are constituted each time a new dataset needs to be analyzed. And indeed, considerable annotation and development efforts have been undertaken to support this latest generation of blood transcriptional modules. This permits us in turn to make available an ecosystem of custom tools and resources that were not available for transcriptional frameworks which have been released before. This includes a compendium of functional profiling reports and reference transcriptional profiles that can be accessed via an interactive web application. In addition, we are releasing another web application for data 
515 browsing and multi-tiered visualization. And it should also be noted that this ecosystem is scalable 516 and will continue to grow over time as more analyses are being conducted, custom scripts 517 developed, and reference dataset profiles added to the annotation scaffold. It is illustrated by 518 ongoing efforts that consist in compiling relevant aggregate-centric information for A28. This is 519 with the intent of improving over time understanding of the clinical relevance and biological 520 significance of this transcriptional signature (accessible by clicking the information icon on the 521 A28 interactive presentation: https://prezi.com/view/E34MhxE5uKoZLWZ3KXjG/ and video 522 demonstration: https://youtu.be/0j-kcE1tlXA). A bioinformatics analysis toolkit that comprises a 523 collection of custom $\mathrm{R}$ scripts has also been developed and made available to the community via 524 GitHub (28). Notably, earlier attempts were made by our group to develop such a support 525 infrastructure in the past, albeit on a smaller scale. But maintaining such resources over long 526 periods of time proved a challenge and these by now have gone offline through a combination of 527 hardware failures, hacking or discontinued institutional support. Such issues should be addressed 528 here in part through adoption of zero cost infrastructure (e.g. hosting of Shiny apps, Prezi 529 presentations or YouTube videos is free as long as public access is maintained).

Finally, the resource that we are making available here has some limitations which are also worth noting. First, our framework is specifically designed to analyze and interpret human blood 532 transcriptome profiling data. Analyses of other tissues or in the context of other species would 533 require the development and use of separate frameworks. Indeed, we also developed fixed modular 534 frameworks for various mouse tissues (29) and in vitro culture systems, including human whole 535 blood (30) and human dendritic cells (31). Second, we generated our transcriptome datasets for 536 module construction using Illumina BeadArrays, a technology that pre-dates RNA sequencing.

537 Our choice to use this technology was based on data availability at our institute, and the decision 
538 to use data that was generated at a single facility over a relatively short period of time. An 539 alternative approach would be to compile available blood RNAseq data that is publicly available 540 and that also comprises appropriately matched controls. The coverage afforded by RNAseq data 541 would likely result in somewhat larger modular gene sets. However, we consider it unlikely that a 542 fundamentally different repertoire would be produced using RNAseq data, as this would assume 543 that entire co-expressed gene sets were missed by microarrays. Third, our modular repertoire is 544 not meant to constitute a ground truth. Indeed, some aggregates are clearly not homogeneous in 545 terms of functionality; and heterogeneity also exists within modules. The attribution of functional 546 annotation titles to modules also confers some degree of subjectivity. However, the repertoire does 547 structure the data so that insights about the biological significance of such a modular signature can 548 be obtained. As a result, while the framework being presented will remain fixed for at least the 549 coming few years, it is likely that the functional annotation map will continue to evolve for the 550 foreseeable future.

551 Going forward, future developments include, firstly, the design and implementation of 552 targeted transcriptional profiling assays based on the current repertoire. Indeed, such repertoires 553 constitute a useful framework for module-based target selection and the development of 554 "Transcriptome Fingerprinting Assays" [a preprint is available: (32)]. Such assays are cost555 effective, robust and scalable. Secondly, fixed transcriptional module repertoires may be leveraged 556 for conducting meta-analysis. It was for instance employed as a basis to perform such an 557 integrative analysis encompassing six independent RSV datasets encompassing 490 unique 558 profiles [a preprint is available: (33)]. More recently, this resource has also been used in a different 559 context to identify signatures associated with CTLA4 and combined CTLA4-PD1 blockade in 560 peripheral blood of ovarian cancer patients [P249: (34)]. It may also be possible to extend the 
561 overall approach described here to develop similar interpretive transcriptomic frameworks for 562 other biological systems.

\section{ACKNOWLEDGEMENTS}

565 The authors would like to thank Quynh-Anh Nguyen, Kimberly O’Brien, Dimitry Popov,

566 Michael Mason, and Cate Speake for technical assistance. We would also like to acknowledge

567 Insight Editing London for assistance in editing the manuscript prior to submission.

568

569

This project has been funded in part with Federal funds from the National Institutes of Health

570 under contract number U01AI082110. Authors affiliated with Sidra Medicine, a member of the

571 Qatar foundation for Education, Science and Community Development, are fully supported by

572 institutional funding. RJW is supported by the Wellcome Trust (203135, 104803), NIH

573 (U01AI115940) and the Francis Crick Institute, which receives funding from the Wellcome Trust

574 (FC10218), CR UK (FC10218) and UKRI (FC10218). Study samples from the CHAVI cohort

575 were obtained through the support from the NIH, NIAID, AI067854 (the Center for HIV/AIDS

576 Vaccine Immunology).

577

578 AUTHOR'S CONTRIBUTIONS

579 Conceptualization: MCA, DR, NB, DC. Data curation: NB, MA. Visualization: DR, MT, DC.

580 Analysis and interpretation: MCA, DR, NB, AAB, EW, MG, BAK, MT, DC. Resources: MT, 581 MA, SP, PK, LC, NJC, AAB, FM, PT, TP, GK, ML, HR, AOG, MB, CB, ML, RJW, CG, GL, $582 \mathrm{MC}, \mathrm{JS}, \mathrm{RT}, \mathrm{FK}, \mathrm{AM}, \mathrm{OR}, \mathrm{KP}, \mathrm{VP}, \mathrm{JB}$. Writing of the first draft: DC. Funding acquisition: GK, 583 KP, VP, OR, JB, DC. Methodology development: MCA, DR, NB, EW. Writing - review \& 
584

585

586

587

588

589

590

591

592

593

594

595

596

597

598

599

600

601

602

603

604

605

editing: MCA, DR, NB, MT, EW, MG, BAK, MA, SP, PK AAB, FM, PT, LC, NJC, JTP, GK, AOG, MB, CB, RJW, CMG, ML, GL, DB, RT, FK, AM, OR, KP, VP, JB, DC. The contributor's roles listed above follow the Contributor Roles Taxonomy (CRediT) managed by The Consortia Advancing Standards in Research Administration Information (CASRAI).

\section{DECLARATION OF INTERESTS}

The authors declare no competing interests

\section{FIGURE LEGENDS}

Figure 1: The module repertoire construction process. A. A collection of 16 transcriptome datasets spanning a wide range of immunological and physiological states were used as input. B. Each dataset was independently clustered via k-means clustering. C. Gene co-clustering events were recorded in a table, where the entries indicate the number of datasets in which co-clustering was observed for a given gene pair. D. The co-clustering table served as the input to a weighted co-clustering graph (see also Supplementary Figure 1), where the nodes represent genes and the edges represent co-clustering events. E. The largest, most highly weighted sub-networks among a large network (here constituting 15,132 nodes) were identified mathematically and assigned a module ID. The genes constituting this module were removed from the selection pool and the process was repeated, resulting in the selection of 382 modules constituted by 14,168 transcripts.

Figure 2: The development of module fingerprint grids. A. The modules were arranged onto the grid as follows: the master set of 382 modules was partitioned into 38 clusters (or aggregates) 
606 based on similarities among their module activity profiles across the sixteen reference datasets

607 (A1-A38). B. A subset of 27 aggregates comprising 2 modules or more in turn occupied a line on

608 the grid. The length of each line was adapted to accommodate the number of modules assigned to

609 each cluster. The format of the grid was fixed for all analyses carried out using this modular

610 framework. C. Changes in transcript abundance at the module level were mapped onto this grid 611 and represented by color spots of varying intensity.

613 Figure 3: Fingerprint grid plots. A. Prototypical fingerprint grid plot: Changes in blood 614 transcript abundance for patients with SLE compared to healthy controls are represented on a 615 fingerprint grid plot for this illustrative use case. The modules occupy a fixed position on the 616 fingerprint grid plots (see Figure 2). An increase in transcript abundance for a given module is 617 represented by a red spot; a decrease in abundance is represented by a blue spot. Modules arranged 618 on a given row belong to a module aggregate (here denoted as A1 to A38). Changes measured at 619 the "aggregate-level" are represented by spots to the left of the grid next to the denomination for 620 the corresponding aggregate. The colors and intensities of the spots are based on the average across 621 each given row of modules. A module annotation grid is provided where a color key indicates the 622 functional associations attributed to some of the modules on the grid (top right). Positions on the 623 annotation grid occupied by modules for which no consensus annotation was attributed are colored 624 white. Positions on the gird for which no modules have been assigned are colored grey. B-D. 625 Fingerprint grid plots for additional reference datasets. 
627 Figure 4: Functional annotation of the transcriptional module repertoire. An interactive 628 application is available to explore the 382 modules comprising the blood transcriptome repertoire. 629 A gene list, along with the ontology, pathway, literature term enrichment and transcriptional 630 profiling data for reference transcriptome datasets (circulating leukocyte populations, 631 hematopoiesis) is provided for each module. Zoom in and out functionalities for close-up 632 examinations of the text and figures embedded in the presentation is possible. Web links providing 633 access to modules within a given aggregate are listed in Table 3. For a demonstration video, please 634 visit: https://youtu.be/8ajESET2mqI.

Figure 5: Individual-level module heatmap. Changes in transcript abundance were determined 637 at the individual level across all modules constituting the repertoire. These changes are represented 638 on a heatmap, where an increase in abundance of a given module is represented in red, and a 639 decrease in abundance is represented in blue. The subjects are organized as columns and the modules as rows. The ordering on the heat map was determined by hierarchical clustering.

643 developed to explore the changes in transcript abundance at the module level across the 16 644 reference datasets used to construct the repertoire. Three types of plot can be displayed and 645 exported: 1) fingerprint grids; 2) module heatmaps displaying changes in abundance in modules 646 comprising a given aggregate across the 16 reference datasets; and 3) module heatmaps displaying 647 changes in abundance in modules comprising a given aggregate across individuals constituting a 648 given dataset. For accessing the application, please visit: 
https://drinchai.shinyapps.io/dc_gen3 module_analysis/\#. For a demonstration video, please visit: https://youtu.be/y_7xKJo5e4.

Figure 7: Module aggregate abundance patterns across the 16 disease or physiological states. A. Patterns of changes in transcript abundance at the aggregate and cohort levels. Each column on the heat map corresponds to a "module aggregate", numbered A1 to A38. Modules A9A14 and A19-A24 were excluded as they each only included one module. Each row on the heatmap corresponds to one of the 16 datasets used to construct the module framework. A red spot on the heatmap indicates an increase in abundance of transcripts comprising a given module cluster for a given disease or physiologic state. A blue spot indicates a decrease in abundance of transcripts. No color indicates no change. Disease or physiological states were arranged based on the level of similarity in the patterns of aggregate activity, determined via hierarchical clustering. B. Representation of the modules and genes constituting aggregate A28. The circle plot represents the six modules constituting aggregate 28 , and the transcripts constituting each of the modules. Some genes on the Illumina BeadArrays can map to multiple probes, which explains the few instances where the same gene can be found in different modules. C. Patterns of changes in transcript abundance at the module-level and gene-level for aggregate A28. The circle plots illustrate the changes at the gene-level for this aggregate for $6 / 16$ datasets. The position of the genes on each of these plots is the same as shown in panel B. Genes for which transcript abundance is changed are shown in red (increase) or in blue (decrease). D. Patterns of changes in transcript abundance at the module and gene level for aggregate A28 in subjects treated with IFNa or IFN $\beta$ treatment The circle plots show changes in abundance for A28 transcripts in patients with 
671 hepatitis C infection treated with IFNa [GSE11342 (16)] or patients with multiple sclerosis treated 672 with IFN $\beta[$ GSE26104 (17)].

673

674 Figure 8: Literature profiles and patterns of changes in abundance across reference datasets 675 for the modules comprising aggregate A28. A. Functional annotation by literature profiling: 676 A portion of a heatmap comprising 382 modules organized as columns, and literature terms 677 organized as rows. The six modules shown are associated with the consensus annotation 678 "Interferon" or "Type 1 Interferon". The clusters of keywords associated with those modules are 679 consistent with this annotation and provide added granularity to the module repertoire for 680 functional profiling and interpretation. B. Changes in abundance across 16 reference datasets: 681 the heatmap (top) represents the changes in abundance of transcripts constituting the six modules 682 comprising aggregate A28 (columns). The modules are functionally associated with interferon 683 responses. The 16 reference datasets are arranged as rows corresponding to different health states.

684 The columns and rows were arranged by hierarchical clustering. Such heatmaps can be accessed 685 and exported for all 16 datasets and 38 module aggregates using the web application: 686 https://drinchai.shinyapps.io/de gen3 module analysis/\# (under the "MODULES x STUDIES" $687 \mathrm{tab})$.

Figure 9: Abundance patterns across individuals. A. Changes in abundance for A28 modules.

690 The heatmaps display the changes in abundance for the same six modules (rows) across individuals

691 (columns) in four reference cohorts. The rows and columns on the heatmap are arranged based on 692 similarities in abundance patterns. B. Changes in abundance for A28 and A35 modules. The 
693 heatmaps display the changes in abundance of six modules constituting aggregate A28 and 21 694 modules constituting aggregate A35 (rows) across individuals (columns) in four reference datasets. 695 Functional annotations associated with different modules is indicated by a color code and 696 corresponding legend. Such heatmaps can be accessed and exported for all 16 datasets and 38 697 module aggregates using the web application:

698 https://drinchai.shinyapps.io/dc_gen3_module_analysis/\# (under the "MODULES X 699 INDIVIDUALS” tab). were used to construct the modular repertoires. These networks factor in differences in coexpression across different "states" of the biological system. For the blood transcriptome, these states would be different diseases or physiological phenotypes. Under Scenario A, the genes are

705

706 707 in weights of 2 and 1 , respectively. co-expressed in all three disease states, so the weight attributed to the edges of the network is three. Under Scenarios B and C, co-clustering only occurs in two or one of the disease states, resulting

Figure S2: Patterns of changes in transcript abundance at the module and gene level for aggregate A28. The large circle plot represents the six modules constituting aggregate 28, and the

711 transcripts constituting each of the modules. Some genes on the Illumina BeadArrays can map to multiple probes, which explains the few instances where the same gene can be found in different modules. Patterns of changes in transcript abundance at the module-level and gene-level for aggregate A28 are shown for all 16 reference datasets. The position of the genes on each of these 
715 plots is fixed. Genes for which transcript abundance is changed are shown in red (increase) or in 716 blue (decrease).

Figure S3: Expression patterns of genes constituting the six A28 modules (rows) across whole by our group [Speake et al. (GSE60424)], and compared the RNAseq transcriptome signatures of

721 six immune-cell subsets and whole blood from patients with various immune-associated diseases

722 (color coding is provided to indicate cell populations and health status). The columns highlighted 723 by the green boxes show the expression levels in patients with MS before and $24 \mathrm{~h}$ after the first 724 dose of IFN- $\beta$.

Figure S4: Abundance patterns of six interferon modules (A28) across individuals from an adult SLE cohort. The abundance levels for six interferon modules belonging to aggregate A28

728 (columns) is shown across a cohort of adult subjects diagnosed with SLE (rows). These subjects 729 were part of the LUPUCE study: the dataset was contributed by our group [Chiche et al. (GEO ID 730 GSE49454)] (11). Changes in transcript abundance were measured in reference to a cohort of 731 healthy subjects included in the study. The colored label with interferon grouping information 732 indicates the classification obtained by Chiche et al. based on three "second generation" modules. 


\section{TABLES}

Table 1: Datasets used for module construction.

741 Sixteen distinct datasets were used as the input for module repertoire construction. Each dataset

742 corresponds to a different condition or physiological state and comprises both cases and matched

743 controls. Each dataset was processed as a single batch at the same facility with the data generated

744 using Illumina HumanHT-12 v3.0 Gene Expression BeadChips. The collection comprises a total

745 of 985 individual transcriptome profiles.

\begin{tabular}{|c|c|c|c|c|c|}
\hline Dataset & Category & Population & $\begin{array}{l}\text { \# Samples } \\
\text { (Cases) }\end{array}$ & $\begin{array}{l}\text { \# Samples } \\
\text { (Control) }\end{array}$ & $\begin{array}{l}\text { \# Samples } \\
\text { (Total) }\end{array}$ \\
\hline $\begin{array}{l}1 \text { Staphylococcus aureus } \\
\text { infection }\end{array}$ & Bacterial Infection & Pediatric & 99 & 44 & 143 \\
\hline 2 Sepsis & Bacterial Infection & Adult & 35 & 12 & 47 \\
\hline 3 Tuberculosis & Bacterial Infection & Adult & 23 & 11 & 34 \\
\hline 4 Influenza & Viral Infection & Pediatric & 25 & 14 & 39 \\
\hline $5 \mathrm{RSV}$ & Viral Infection & Pediatric & 70 & 14 & 84 \\
\hline $6 \mathrm{HIV}$ & Viral Infection & Adult & 28 & 35 & 63 \\
\hline $\begin{array}{l}7 \text { Systemic Lupus } \\
\text { Erythematosus }\end{array}$ & Autoimmune & Pediatric & 55 & 14 & 69 \\
\hline 8 Multiple Sclerosis & Autoimmune & Adult & 34 & 22 & 56 \\
\hline 9 Juvenile Dermatomyositis & Autoimmune & Pediatric & 40 & 9 & 49 \\
\hline 10 Kawasaki disease & Autoinflammatory & Pediatric & 21 & 23 & 44 \\
\hline $\begin{array}{l}11 \text { Systemic Onset Idiopathic } \\
\text { Arthritis }\end{array}$ & Autoinflammatory & Pediatric & 62 & 23 & 85 \\
\hline 12 COPD & Inflammatory & Adult & 19 & 24 & 43 \\
\hline 13 Melanoma & Malignancy & Adult & 22 & 5 & 27 \\
\hline 14 Pregnancy & Physiologic variant & Adult & 25 & 20 & 45 \\
\hline 15 Liver transplant recipients & Immunosuppressed & Adult & 94 & 30 & 124 \\
\hline 16 B-cell deficiency & Immunodeficiency & Adult & 20 & 13 & 33 \\
\hline
\end{tabular}


Table 2: The characteristics of the input datasets used to construct three consecutive generations of blood transcriptome module repertoires.

\begin{tabular}{|c|c|c|c|}
\hline & Generation 1 & Generation 2 & Generation 3 \\
\hline Number of States & 8 & 7 & 16 \\
\hline \multicolumn{4}{|l|}{ States } \\
\hline $\begin{array}{l}\text { Systemic onset juvenile idiopathic } \\
\text { arthritis }\end{array}$ & $\mathrm{X}$ & $X$ & $\mathrm{X}$ \\
\hline Systemic lupus erythematosus & X & $\mathrm{X}$ & $\mathrm{X}$ \\
\hline Juvenile dermatomyositis & & & $\mathrm{X}$ \\
\hline Type 1 Diabetes & X & & \\
\hline Multiple sclerosis & & & $\mathrm{x}$ \\
\hline Kawasaki disease & & & $\mathrm{X}$ \\
\hline COPD & & & $\mathrm{X}$ \\
\hline Tuberculosis & & X & $X$ \\
\hline Sepsis & & $\mathrm{x}$ & X \\
\hline Respiratory Syncytial Virus infection & & & $\mathrm{X}$ \\
\hline Influenza virus infection & X & & $\mathrm{X}$ \\
\hline $\begin{array}{l}\text { Human Immunodeficiency Virus } \\
\text { infection }\end{array}$ & & $x$ & $\mathrm{X}$ \\
\hline Escherichia coli infection & $X$ & & \\
\hline Staphylococcus aureus infection & X & & $\mathrm{X}$ \\
\hline B-cell deficiency & & & $\mathrm{X}$ \\
\hline Liver transplant recipients & $\mathrm{X}$ & $x$ & $\mathrm{X}$ \\
\hline Metastatic melanoma & $\mathrm{X}$ & $\mathrm{x}$ & X \\
\hline Pregnancy & & & $\mathrm{X}$ \\
\hline Number of input datasets & 8 & 9 & 16 \\
\hline Number of individual profiles & 239 & 410 & 985 \\
\hline Sample source & PBMCs & Whole Blood & Whole Blood \\
\hline Platform & Affymetrix U133A\&B & Illumina Hu6 v2 & Illumina HT12 v3.0 \\
\hline Rounds of module selection & 3 & 8 & 15 \\
\hline Number of modules & 28 & 260 & 382 \\
\hline Year published \& reference & $2008(4)$ & $2013(3)$ & Current work \\
\hline
\end{tabular}


761 Table 3: Links to module aggregates annotation pages

\begin{tabular}{|c|c|c|}
\hline Aggregate & Function & Links \\
\hline A1 & Lymphocytic & https://prezi.com/view/sxap39tKxkmCNTTNIIVO/ \\
\hline A2 & TBD & https://prezi.com/view/96GWajx5mZjuRS4B6gjA/ \\
\hline A3 & TBD & https://prezi.com/view/OWFVI51FNDOWWwNgsgJZ/ \\
\hline A4 & TBD & https://prezi.com/view/2Zbq8ZDYbO4hbUd4r2KF/ \\
\hline A5 & Lymphocytic & https://prezi.com/view/62tgA5E6roOlk5DRNvS1/ \\
\hline A6 & Lymphocytic & https://prezi.com/view/Uks2Nd4lvizNNFVPtBEy/ \\
\hline A7 & TBD & https://prezi.com/view/kKfergNjOSkLXyFtm0Dg/ \\
\hline A8 & TBD & https://prezi.com/view/Y4uk1RPJyNcSndJYnFX6/ \\
\hline A9 & TBD & https://prezi.com/view/jgYehQ9QhyADAttEsdol/ \\
\hline A15 & TBD & https://prezi.com/view/jgYehQ9QhyADAttEsdol/ \\
\hline A16 & TBD & https://prezi.com/view/SKzHeAOXYdLYvy2sY8gP/ \\
\hline A17 & TBD & https://prezi.com/view/FS7sE1Vqew5g8EKOM1AM/ \\
\hline A18 & TBD & https://prezi.com/view/aZMLfIMNVrV7JnValILm/ \\
\hline A24 & Oxidative phosphorylation & https://prezi.com/view/eiXvf2LNBLFRgrtaeCuM/ \\
\hline A25 & TBD & https://prezi.com/view/pwyojaU62Z7GT102ZYwM/ \\
\hline A26 & TBD & https://prezi.com/view/9CErpW3NwpN2HgRS3Hzf/ \\
\hline A27 & Cell cycle & https://prezi.com/view/GgliA0K9kSFHbpVj2185/ \\
\hline A28 & Interferon & https://prezi.com/view/E34MhxE5uKoZLWZ3KXjG/ \\
\hline A29 & TBD & https://prezi.com/view/W4TShTd32dEJxOXPOF1U/ \\
\hline A30 & TBD & https://prezi.com/view/kl7VHoJTWug0sn7TgXut/ \\
\hline A31 & TBD & https://prezi.com/view/GqtUO22JJISf16zMJKbB/ \\
\hline A32 & TBD & https://prezi.com/view/qlbG9VFzegOndQKD8aiy/ \\
\hline A33 & Inflammation & https://prezi.com/view/VBqKqHuLWCra3OJOIZRR/ \\
\hline A34 & TBD & https://prezi.com/view/HcSgIEGP3TJjTSpaPCxv/ \\
\hline A35 & Inflammation & https://prezi.com/view/7Q20FyW6Hrs5NjMaTUyW/ \\
\hline A36 & Erythroid & https://prezi.com/view/M7dnzt|2h61gXrKFQeB2/ \\
\hline A37 & Erythroid & https://prezi.com/view/YyQs4WiXSNfOYXE79lfS/ \\
\hline A38 & Erythroid & https://prezi.com/view/OKUIPICKUZGeUjb206R5/ \\
\hline
\end{tabular}


763

764

765

766

767

768

769

770

771

772

773

774

775

776

777

778

779

780

781

782

783

784

785

\section{METHODS:}

\section{Study subjects}

Gene expression datasets from 985 de-identified subjects from distinct cohorts were used for this work. Written informed consent was obtained from all participants. Studies were approved by Institutional Review Boards of the Baylor College of Medicine (COPD dataset: H-18029), the University of Texas Southwestern Medical Center and Baylor Health Care System (Influenza, RSV, S. aureus and Kawasaki disease datasets: UTSW \#0802-447 / BIIR \#002-141), Saint Jude's Research Hospital (B-cell deficiency), the Baylor Health Care System (Liver transplant: 002-197, Pregnancy: 009-257, Multiple sclerosis: 009-240, Melanoma: 006-025 \& 097-027), Khon Kaen University (Sepsis), the University of Texas Southwestern Medical Center (SoJIA, Dermatomyositis, SLE), Duke University and the Baylor Health Care System (HIV: Duke 848506-4R0 / Baylor 006-177), St. Mary's Hospital London, UK and University of Cape Town, Cape Town, Republic of South Africa (Tuberculosis: St Mary’s REC 06/Q0403/128, U of Cape Town REC 012/2007). The gene expression datasets were selected to cover major classes of immune states (Table 2), were required to have at least 25 samples in total, and at least $20 \%$ of the total samples were required to be appropriately matched controls.

General descriptions of the study cohorts are as follows: S. aureus cohort: Children with community-acquired S. aureus infection were enrolled. The clinical syndromes of these patients included skin and soft tissue infection, bacteremia, osteomyelitis, suppurative arthritis, pyomyositis, pneumonia, and disseminated disease. Patients diagnosed with toxic shock syndrome, polymicrobial infections, or treated with corticosteroids in the preceding four weeks were excluded. Sepsis cohort: Diagnosis of sepsis was based on accepted international guidelines 
and defined as presentation with two or more of the following criteria for the systemic inflammatory response syndrome: fever (temperature $>38^{\circ} \mathrm{C}$ or $<36^{\circ} \mathrm{C}$ ), tachycardia (heart rate $>90$ beats/minute), leukocytosis or leukocytopenia (white blood cell count $\geq 12 \times 109 / 1$ or $\leq 4 \times$ 109/1). Blood was collected within 24 hours following the diagnosis of sepsis. Samples were selected for microarray analysis from subjects who had the diagnosis of bacteremic sepsis retrospectively confirmed by the isolation of a causative organism on blood culture. TB cohort: Patients were prospectively recruited and sampled, before any anti-mycobacterial treatment was initiated. Active TB disease was confirmed by laboratory isolation of M. tuberculosis on mycobacterial culture of a respiratory specimen (either sputum or bronchoalvelolar lavage fluid). Influenza cohort: Children with confirmed influenza infection were recruited. Those with documented bacterial co-infections, children with chronic conditions and systemic steroid treatment within 2 weeks before enrollment were excluded. RSV cohort: Patients with confirmed RSV infection were recruited. Children with documented bacterial co-infections, congenital heart disease, chronic lung disease, immunodeficiency, prematurity $(<36 \mathrm{wk})$, systemic steroid treatment within 2 weeks before presentation or additional chronic comorbidities were excluded. HIV cohort: Blood samples were obtained from adult patients diagnosed with HIV infection. At enrollment patients were verified as acute Fiebig stages 4 to 6 (plasma RNA+, third generation EIA+, Western blot indeterminant or + ). SLE cohort: Blood samples were obtained from pediatric patients diagnosed with Systemic Lupus Erythematosus and healthy controls matched for demographic characteristics. MS cohort: Subjects enrolled in the MS cohort had an established diagnosis of relapsing-remitting multiple sclerosis (MS), separately confirmed by an experienced MS neurologist (JTP), exhibited no other health conditions, and had received no treatment(s) for MS, including corticosteroids, for at least 3 months prior to blood draw. Juvenile 
Dermatomyositis cohort: Blood samples were obtained from pediatric patients diagnosed with juvenile dermatomyositis and healthy controls matched for demographic characteristics. Kawasaki Disease cohort: Subjects $<18$ years of age who met the definition of Kawasaki Disease based on the American Heart Association (AHA) criteria (35) were enrolled alongside age and gender matched healthy controls. Systemic onset Juvenile Arthritis cohort: Blood samples were obtained from SoJIA patients displaying systemic symptoms only (fever, rash, and/or pericarditis) or displaying systemic symptoms accompanied by arthritis. COPD cohort: Enrollment criteria included age over 40 years, no history of concurrent lung cancer, chest surgery, or chronic lung diseases other than COPD (e.g., sarcoidosis, fibrosis, etc.). Participants had no history of allergies or asthma and at the time of initial recruitment had not received oral or systemic corticosteroids during the previous 6 weeks; volunteers were enrolled from three clinics within the Texas Medical Center in Houston, Texas. B-cell deficiency cohort: This cohort comprised adults with diagnosis of XLA as documented by markedly reduced numbers of peripheral blood B cells. Pregnancy cohort: Pregnant women were recruited at the Baylor Institute for Immunology Research (Dallas, TX) for a study of immunological signatures of pregnancy. Melanoma cohort: Enrollment criteria included ages of 21-75 years stage M1a, M1b, M1c biopsy proven metastatic melanoma patients with measurable metastatic lesions by physical exam or scans, acceptable $\mathrm{CBC}$ and blood chemistry results, adequate hepatic and renal function, no active CNS metastatic disease. Liver transplant cohort: Enrollment criteria included age 17-65 years, having received a liver transplant under maintenance immunosuppression therapy. Subjects in this cohort had not received an acute or chronic rejection diagnosis at the time of sampling. 


\section{RNA extraction and processing}

832 Whole blood for all sample sets were collected into Tempus Blood RNA Tubes (Thermo Fisher

833 Scientific). Total RNA was isolated from whole blood lysate using a MagMAX for Stabilized 834 Blood Tubes RNA Isolation Kit for Tempus Blood RNA Tubes (Thermo Fisher Scientific). RNA quality and quantity were assessed using an Agilent 2100 Bioanalyzer (Agilent Technologies) and

836 a NanoDrop 1000 (NanoDrop Products, Thermo Fisher Scientific). Samples with RNA integrity 837 numbers values $>6$ were retained for further processing.

\section{Microarray analysis and data preprocessing}

839 Gene expression profiles from whole blood samples generated using Illumina HumanHT-12 v3.0 840 or Illumina HumanHT-12 v4.0 expression BeadChips were obtained from 16 groups of patients 841 and controls selected as above. Thus, 16 datasets were used as the input (Table 1). The expression 842 data for each dataset were preprocessed and independently clustered. First, the probes were 843 discarded if they were not present (detection $P<0.01$ ) in at least 10 samples or in at least $10 \%$ of 844 the samples, whichever was greater. Then, the sample data for each dataset were normalized using 845 the BeadStudio average normalization algorithm. Once normalized, the signal was floored such 846 that all signals $<10$ were set to 10 . Then, the fold change was calculated relative to the median 847 signal for that probe across all samples. If the difference between a signal and the probe's median 848 signal was $<30$, or the calculated absolute magnitude of the fold change was $<1.2$, the fold change 849 was set to 1 to reduce the noise from low-level responses. At this stage, the probes were filtered 850 again. Probes were only retained if they had a calculated absolute fold change $>1$ in at least 10 851 samples or in at least $10 \%$ of the samples, whichever was greater. Finally, the data was transformed 852 to the $\log _{2}$ of the calculated fold changes. 


\section{Module construction algorithm}

854 Sets of coordinately regulated genes, or transcriptional modules, were extracted from the patient's 855 whole blood microarray datasets. Each of the preprocessed microarray datasets were clustered in 856 parallel using Euclidean distance and the Hartigan's K-Means clustering algorithm. The 'ideal'

857 number of clusters $(\mathrm{k})$ for each dataset was determined within a range of $\mathrm{k}=1-100$ by means of the 858 jump statistic (36). Taking the 16 sets of clusters as the input (Table 2), a weighted co-cluster 859 graph was constructed (Chaussabel, Quinn et al. 2008, Chaussabel and Baldwin 2014). To select 860 modules, an iterative algorithm was used to extract the sets of probes that are most frequently 861 clustered together in the same datasets, proceeding from the most stringent requirements to the 862 least, as previously described (Chaussabel, Quinn et al. 2008). This iteration differed from the 863 previous implementation of this algorithm in that the $\mathrm{k}$ value was calculated independently for 864 each dataset cluster and the size of the core sub-networks was smaller (10 probes). The algorithm 865 also differed from previous implementations to ensure that the core sub-networks co-clustered in 866 the same datasets. Further details and an example of the code are included in the supplemental 867 methods (Supplementary File 1). The resulting 382 module set constitutes the third generation of 868 modular blood transcriptome repertoire constructed since the development of the first generation 869 published in 2008 (4), and the second generation published in 2013 (3).

\section{Module annotation}

871 Gene Ontology / Pathway enrichment: Module gene lists were investigated using Database for 872 Annotation Visualization and Integrated Discovery (DAVID) version 6.7 (12). This database uses 873 a modified Fisher exact test to identify specific biological/functional categories that are 874 overrepresented in gene sets in comparison with a reference set. The top matched DAVID 875 annotation cluster (using default settings), the top matched canonical pathway from Kyoto 
876

877

878

879

880

881

882

883

884

885

886

887

888

889

890

891

892

893

894

895

896

897

898

encyclopedia of genes and genomes (KEGG), the top matched pathway from BioCarta, and the top matched Gene Ontology biologic process (GO_BP) and molecular function (GO_MF) terms were identified for each module. Each module was also investigated for significant overlap with two other established blood transcriptome module repertoires $(3,9)$. The findings are summarized in the module annotation spreadsheet (Supplemental File 2).

Gene Set Annotation (GSAn): To further annotate the modules, a new alternative to statistical enrichment analysis tool called GSAn was applied (13). Statistical enrichment methods may have limitations (36-38), as these methods tend to focus on the subpart of the most studied genes and to provide gene set annotation results that cover a limited number of the well-annotated genes. To address these issues, GSAn offers: (i) an original method that combines semantic similarity measures and data mining approaches to perform a unified and synthetic annotation for a gene set of interest, and (ii) a visualization approach to facilitate the interactive exploration of the gene set annotation results according to the hierarchical structure of Gene Ontology (13). The tool is available online: https://gsan.labri.fr/. A page listing analysis results for all 382 generation 3 blood transcriptome modules can be accessed here: https://ayllonbe.github.io/modulesV3/index.html.

Pathway enrichment analyses: These analyses were conducted using Ingenuity Pathway Analysis (Qiagen, Valencia, CA).

Literature profiling: Literature Lab ${ }^{\mathrm{TM}}$ (LitLab; from Acumenta Biotech, Boston, MA) was used to associate genes within a particular module to terms used in PubMed abstracts (Febbo, Mulligan et al. 2007). Association scores reflecting the strength of the associations were used to calculate the "Product Scores". The top three terms that showed the strongest association and 
highest "Product Scores" were used to create the functional annotation. A similar approach using LitLab has been previously reported (Alsina, Israelsson et al. 2014). The steps taken to annotate

901 all 382 modules is described here in brief. All statistical analyses were performed using

902 Microsoft Excel (2010) with Visual Basic for Applications (VBA), Linux-based command line

903 in Mac OS, and R statistical software.

904 To construct a Product Scores Table, all the terms available in LitLab $(>80,000)$ were 905 listed. Then, the genes in each module were submitted as a list to LitLab Editor and manually 906 validated using LitLab's built-in validation tool and/or NCBI Gene 907 (https://www.ncbi.nlm.nih.gov/gene) prior to submission for analysis using all domains available.

908 After completing the analysis, the summary result page was exported to an xls file. Using the 909 UNIX command line, the exported files were converted to csv files with the filename appended in 910 the last column of each row and vertically appended. The "merged" file was used to populate the 911 table that included all the available LitLab terms. The top three terms with the highest Product 912 Scores were selected to represent the module functional annotation and are tabulated in column I 913 of the module annotation table (Supplementary file 2).

\section{$915 \quad$ Fingerprint grid plot visualization}

916 Modules were arranged on a grid based on their similarities in patterns of activity across the 16 917 input datasets, each of them corresponding to a different pathological or physiological state. First, 918 the modules were partitioned using k-means clustering, which resulted in the constitution of 38 919 clusters. Given the possibility of collapsing values of the modules constituting each cluster in a 920 single "aggregate" value, the term "module aggregate" was used to designate each cluster (A1 to 921 A38). Of these $38 \mathrm{k}$-means clusters, 27 comprised $>1$ module. The modules were then arranged 
922

923

924 of modules for a given aggregate (42 for aggregate A2). For each module, the highest of the two

925 values indicating an increase or a decrease was selected for visualization (e.g. if \% increase $>\%$ 926 decrease, then a red sport representing \% increase is shown).

927

928

929

930

931

932

933

934

935

936

937

938

939

940

941

942

943

944

on a grid with each row corresponding to modules belonging to the same aggregate (Figure 4). The total number of rows on the grid equaled 27 and number of columns equaled the largest number values indicating an increase or a decrease was selected for visualization (e.g. if \% increase $>\%$

\section{Module Fingerprint analysis and visualization}

The modular analysis was performed by using 14,168 transcripts. Fold change was computed using gene expression data prior $\log 2$ transformation. For group comparisons, paired t-test was performed on $\log 2$-transformed data (Fold change $(\mathrm{FC})$ cut off $=1.5$; FDR cut off $=0.1)$. For individual-patients analysis, each sample was compared to mean of control samples in each dataset. Cut off comprised a absolute $\mathrm{FC}>1.5$ and a difference in gene expression level $>10$. A module was considered to be "responsive" when the proportion of differentially expressed transcripts (as defined above) was greater than 15\%. Data visualization were performed using “ComplexHeatmap” (37).

\section{Generation of Circular Plots}

Circular plots were generated to represent module expression at the gene-level (Figure 7). Fold change in case vs control group in each dataset were shown. Probes that passed FDR $<0.1$ and FC $>1.5$ were present in gradated red colors and those with $\mathrm{FC}<-1.5$ were represented in gradated blue colors. The position of the genes on each of the circular plots is fixed. 


\section{Statistical analyses}

946 Numerical data were processed and analyzed using R statistical software (version1.1.463-C2009-

9472018 RStudio, Inc). Student's t-test was used to determine which group was different. Values of

$948 p<0.05$ were considered to indicate statistical significance, with adjustment by multiple-testing

949 correction when needed (FDR, Benjamini-Hochberg procedure).

950

951 Availability of data and material

952 Transcriptome profiling data are deposited in the Gene Expression Omnibus, 953 https://www.ncbi.nlm.nih.gov/geo/, under the accession GSE100150.

954

955 SUPPLEMENTAL INFORMATION

956 Supplementary File 1: Module generation method and pseudocode

957 This Word document describes the algorithm used to construct this modular repertoire framework

958 along with pseudocode, which may be used as a basis for implementation in programming

959 languages such as R or Python.

960 Supplementary File 2: Annotated module repertoire

961 This Excel spreadsheet lists the 382 modules constituting this third generation of blood 962 transcriptome module repertoire. Included are the number of genes, the list of member genes by 963 symbol and the probe ID, summarized functional annotations.

964 Supplementary File 3: Module fingerprints of all 16 pathological and physiological states

965 This PDF file contains the modular fingerprints generated for each of the 16 input datasets. 966 


\section{REFERENCES}

968

969

970

971

972

973

974

975

976

977

978

979

980

981

982

983

984

985

986

987

988

989

990

991

992

993

994

995

996

997

998

999

1000

1001

1. Hulsen T, Jamuar SS, Moody AR, Karnes JH, Varga O, Hedensted S, et al. From Big Data to Precision Medicine. Front Med. 2019;6:34.

2. Chaussabel D, Baldwin N. Democratizing systems immunology with modular transcriptional repertoire analyses. Nat Rev Immunol. 2014;14(4):271-80.

3. Obermoser G, Presnell S, Domico K, Xu H, Wang Y, Anguiano E, et al. Systems scale interactive exploration reveals quantitative and qualitative differences in response to influenza and pneumococcal vaccines. Immunity. 2013 Apr 18;38(4):831-44.

4. Chaussabel D, Quinn C, Shen J, Patel P, Glaser C, Baldwin N, et al. A modular analysis framework for blood genomics studies: application to systemic lupus erythematosus. Immunity. $2008 \mathrm{Jul}$ 18;29(1):150-64.

5. Oswald M, Curran ME, Lamberth SL, Townsend RM, Hamilton JD, Chernoff DN, et al. Modular analysis of peripheral blood gene expression in rheumatoid arthritis captures reproducible gene expression changes in tumor necrosis factor responders. Arthritis Rheumatol Hoboken NJ. 2015 Feb;67(2):344-51.

6. Berry MPR, Graham CM, McNab FW, Xu Z, Bloch SAA, Oni T, et al. An interferon-inducible neutrophil-driven blood transcriptional signature in human tuberculosis. Nature. 2010 Aug 19;466(7309):973-7.

7. Banchereau R, Hong S, Cantarel B, Baldwin N, Baisch J, Edens M, et al. Personalized Immunomonitoring Uncovers Molecular Networks that Stratify Lupus Patients. Cell. $2016 \mathrm{Apr}$ 21;165(3):551-65.

8. Pankla R, Buddhisa S, Berry M, Blankenship DM, Bancroft GJ, Banchereau J, et al. Genomic transcriptional profiling identifies a candidate blood biomarker signature for the diagnosis of septicemic melioidosis. Genome Biol. 2009;10(11):R127.

9. Li S, Rouphael N, Duraisingham S, Romero-Steiner S, Presnell S, Davis C, et al. Molecular signatures of antibody responses derived from a systems biology study of five human vaccines. Nat Immunol. 2014 Feb;15(2):195-204.

10. Zhou W, Altman RB. Data-driven human transcriptomic modules determined by independent component analysis. BMC Bioinformatics. 2018 Sep 17;19(1):327.

11. Chiche L, Jourde-Chiche N, Whalen E, Presnell S, Gersuk V, Dang K, et al. Modular transcriptional repertoire analyses of adults with systemic lupus erythematosus reveal distinct type I and type II interferon signatures. Arthritis Rheumatol Hoboken NJ. 2014 Jun;66(6):1583-95.

12. Jiao X, Sherman BT, Huang DW, Stephens R, Baseler MW, Lane HC, et al. DAVID-WS: a stateful web service to facilitate gene/protein list analysis. Bioinforma Oxf Engl. 2012 Jul 1;28(13):1805-6. 
1002

1003

1004

1005

1006

1007

1008

1009

1010

1011

1012

1013

1014

1015

1016

1017

1018

1019

1020

1021

1022

1023

1024

1025

1026

1027

1028

1029

1030

1031

1032

1033

1034

1035

1036

1037

13. Ayllon-Benitez A, Bourqui R, Thébault P, Mougin F. GSAn: an alternative to enrichment analysis for annotating gene sets. NAR Genomics Bioinforma [Internet]. 2020 Jun 1 [cited 2020 Apr 1];2(2). Available from: https://academic.oup.com/nargab/article/2/2/lqaa017/5805305

14. Novershtern N, Subramanian A, Lawton LN, Mak RH, Haining WN, McConkey ME, et al. Densely interconnected transcriptional circuits control cell states in human hematopoiesis. Cell. 2011 Jan 21;144(2):296-309.

15. Linsley PS, Speake C, Whalen E, Chaussabel D. Copy number loss of the interferon gene cluster in melanomas is linked to reduced $T$ cell infiltrate and poor patient prognosis. PloS One. 2014;9(10):e109760.

16. Taylor MW, Tsukahara T, McClintick JN, Edenberg HJ, Kwo P. Cyclic changes in gene expression induced by Peg-interferon alfa-2b plus ribavirin in peripheral blood monocytes (PBMC) of hepatitis C patients during the first 10 weeks of treatment. J Transl Med. 2008 Nov 5;6:66.

17. Malhotra S, Bustamante MF, Pérez-Miralles F, Rio J, Ruiz de Villa MC, Vegas E, et al. Search for specific biomarkers of IFN $\beta$ bioactivity in patients with multiple sclerosis. PloS One.

2011;6(8):e23634.

18. Bennett L, Palucka AK, Arce E, Cantrell V, Borvak J, Banchereau J, et al. Interferon and granulopoiesis signatures in systemic lupus erythematosus blood. J Exp Med. 2003 Mar 17;197(6):711-23.

19. Baechler EC, Batliwalla FM, Karypis G, Gaffney PM, Ortmann WA, Espe KJ, et al. Interferoninducible gene expression signature in peripheral blood cells of patients with severe lupus. Proc Natl Acad Sci U S A. 2003 Mar 4;100(5):2610-5.

20. Spann KM, Tran K-C, Chi B, Rabin RL, Collins PL. Suppression of the induction of alpha, beta, and lambda interferons by the NS1 and NS2 proteins of human respiratory syncytial virus in human epithelial cells and macrophages [corrected]. J Virol. 2004 Apr;78(8):4363-9.

21. Kalinowski A, Galen BT, Ueki IF, Sun Y, Mulenos A, Osafo-Addo A, et al. Respiratory syncytial virus activates epidermal growth factor receptor to suppress interferon regulatory factor 1-dependent interferon-lambda and antiviral defense in airway epithelium. Mucosal Immunol. 2018;11(3):95867.

22. Carretero R, Wang E, Rodriguez Al, Reinboth J, Ascierto ML, Engle AM, et al. Regression of melanoma metastases after immunotherapy is associated with activation of antigen presentation and interferon-mediated rejection genes. Int J Cancer J Int Cancer. 2012 Jul 15;131(2):387-95.

23. Critchley-Thorne RJ, Simons DL, Yan N, Miyahira AK, Dirbas FM, Johnson DL, et al. Impaired interferon signaling is a common immune defect in human cancer. Proc Natl Acad Sci U S A. 2009 Jun 2;106(22):9010-5.

24. Baechler EC, Bilgic H, Reed AM. Type I interferon pathway in adult and juvenile dermatomyositis. Arthritis Res Ther. 2011;13(6):249. 
1038

1039

1040

1041

1042

1043

1044

1045

1046

1047

1048

1049

1050

1051

1052

1053

1054

1055

1056

1057

1058

1059

1060

1061

1062

1063

1064

1065

1066

1067

1068

1069

1070

1071

1072

1073

1074

25. Subramanian A, Tamayo P, Mootha VK, Mukherjee S, Ebert BL, Gillette MA, et al. Gene set enrichment analysis: a knowledge-based approach for interpreting genome-wide expression profiles. Proc Natl Acad Sci U S A. 2005 Oct 25;102(43):15545-50.

26. Godec J, Tan Y, Liberzon A, Tamayo P, Bhattacharya S, Butte AJ, et al. Compendium of Immune Signatures Identifies Conserved and Species-Specific Biology in Response to Inflammation. Immunity. 2016 Jan 19;44(1):194-206.

27. Altman MC, Gill MA, Whalen E, Babineau DC, Shao B, Liu AH, et al. Transcriptome networks identify mechanisms of viral and nonviral asthma exacerbations in children. Nat Immunol. 2019;20(5):637-51.

28. Rinchai D, Roelands J, Hendrickx W, Altman MC, Bedognetti D, Chaussabel D. Blood transcriptional module repertoire analysis and visualization using R. bioRxiv. 2020 Jul 16;2020.07.16.205963.

29. Singhania A, Graham CM, Gabryšová L, Moreira-Teixeira L, Stavropoulos E, Pitt JM, et al. Transcriptional profiling unveils type I and II interferon networks in blood and tissues across diseases. Nat Commun. 2019 28;10(1):2887.

30. Alsina L, Israelsson E, Altman MC, Dang KK, Ghandil P, Israel L, et al. A narrow repertoire of transcriptional modules responsive to pyogenic bacteria is impaired in patients carrying loss-offunction mutations in MYD88 or IRAK4. Nat Immunol. 2014 Dec;15(12):1134-42.

31. Banchereau R, Baldwin N, Cepika A-M, Athale S, Xue Y, Yu Cl, et al. Transcriptional specialization of human dendritic cell subsets in response to microbial vaccines. Nat Commun. 2014 Oct 22;5:5283.

32. Altman MC, Baldwin N, Whalen E, al-shaikhly, taha, Presnell S, Khaenam P, et al. A Transcriptome Fingerprinting Assay for Clinical Immune Monitoring. bioRxiv [Internet]. Available from: https://www.biorxiv.org/content/10.1101/587295v1

33. Rinchai D, Konza O, Hassler S, Martina F, Mejias A, Ramilo O, et al. Characterizing blood modular transcriptional repertoire perturbations in patients with RSV infection: a hands-on workshop using public datasets as a source of training material. bioRxiv. 2019 Jan 22;527812.

34. 34th Annual Meeting \& Pre-Conference Programs of the Society for Immunotherapy of Cancer (SITC 2019): part 1 : National Harbor, MD, USA. 6-10 November 2019. J Immunother Cancer. 2019 Nov 6;7(Suppl 1):282.

35. Newburger JW, Takahashi M, Gerber MA, Gewitz MH, Tani LY, Burns JC, et al. Diagnosis, Treatment, and Long-Term Management of Kawasaki Disease: A Statement for Health Professionals From the Committee on Rheumatic Fever, Endocarditis and Kawasaki Disease, Council on Cardiovascular Disease in the Young, American Heart Association. Circulation. 2004 Oct 26;110(17):2747-71.

36. Sugar CA, James GM. Finding the Number of Clusters in a Dataset: An Information-Theoretic Approach. J Am Stat Assoc. 2003;98(463):750-63.

37. Gu Z, Eils R, Schlesner M. Complex heatmaps reveal patterns and correlations in multidimensional genomic data. Bioinforma Oxf Engl. 2016 15;32(18):2847-9. 

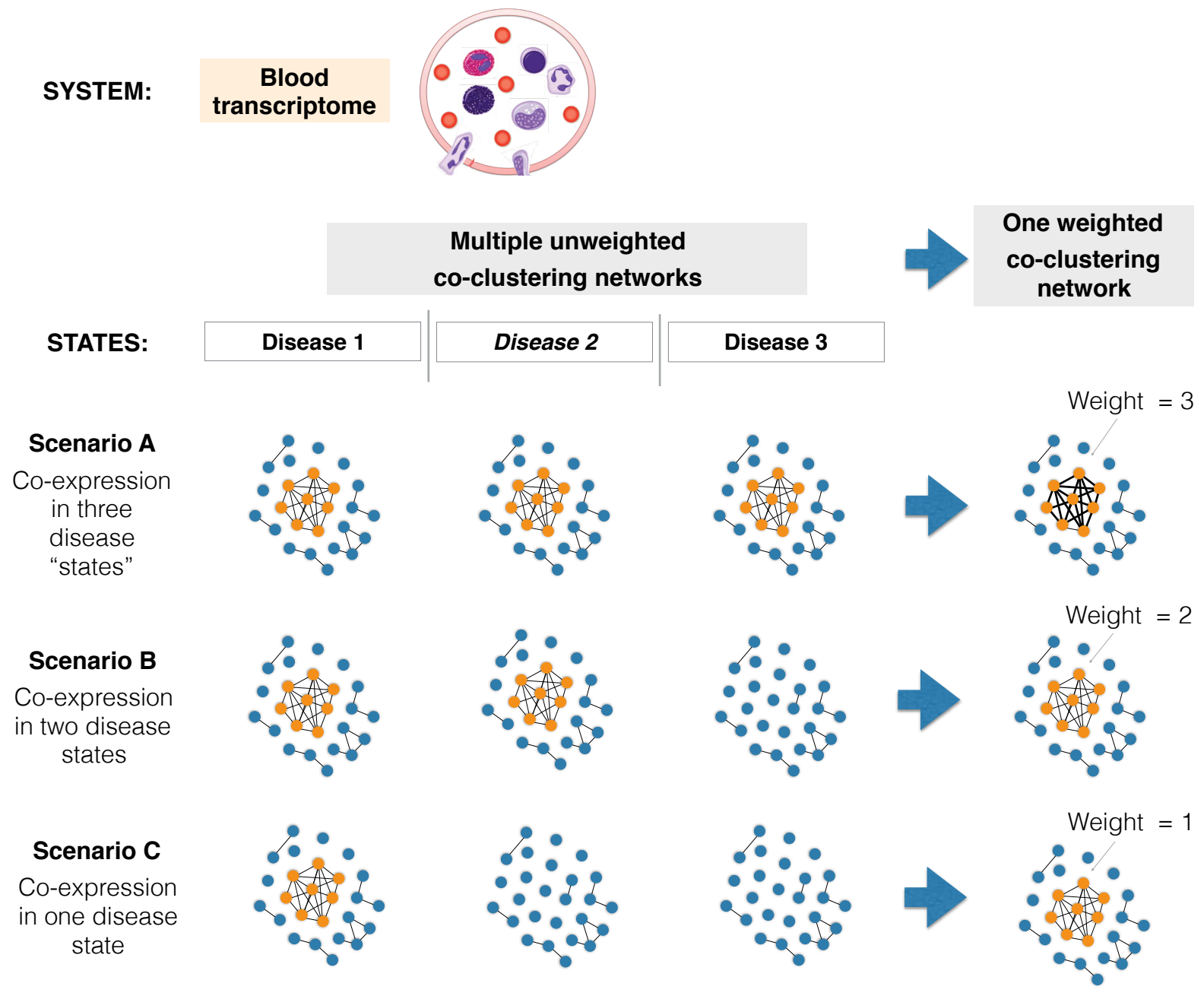

Figure S1: Construction of weighted co-clustering networks. Weighted co-clustering networks were used to construct the modular repertoires. These networks factor in differences in co-expression across different "states" of the biological system. For the blood transcriptome, these states would be different diseases or physiological phenotypes. Under Scenario A, the genes are co-expressed in all three disease states, so the weight attributed to the edges of the network is three. Under Scenarios B and C, coclustering only occurs in two or one of the disease states, resulting in weights of 2 and 1, respectively. 

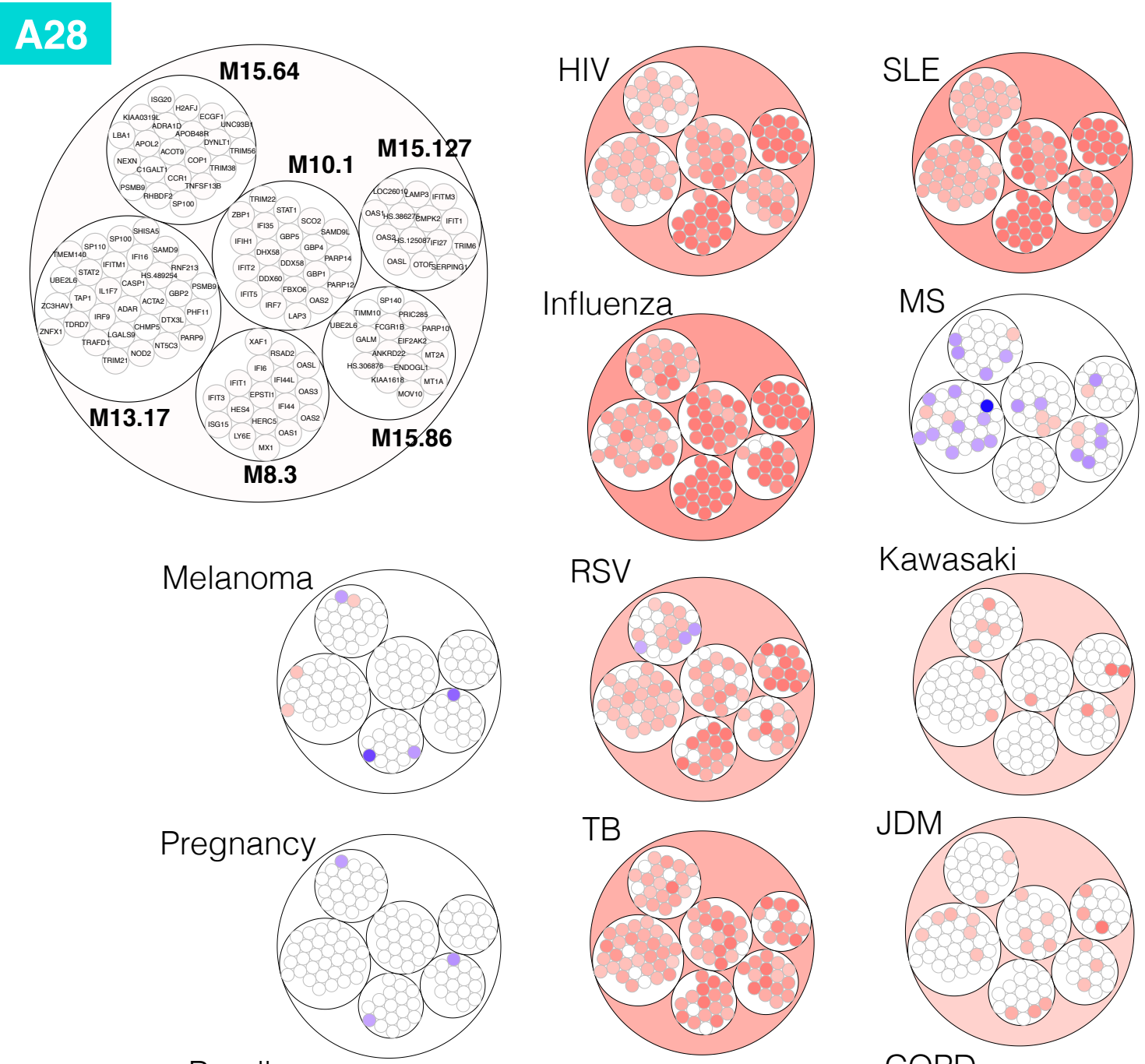

B-cell

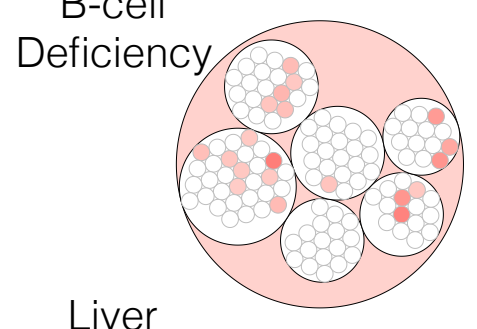

Sepsis
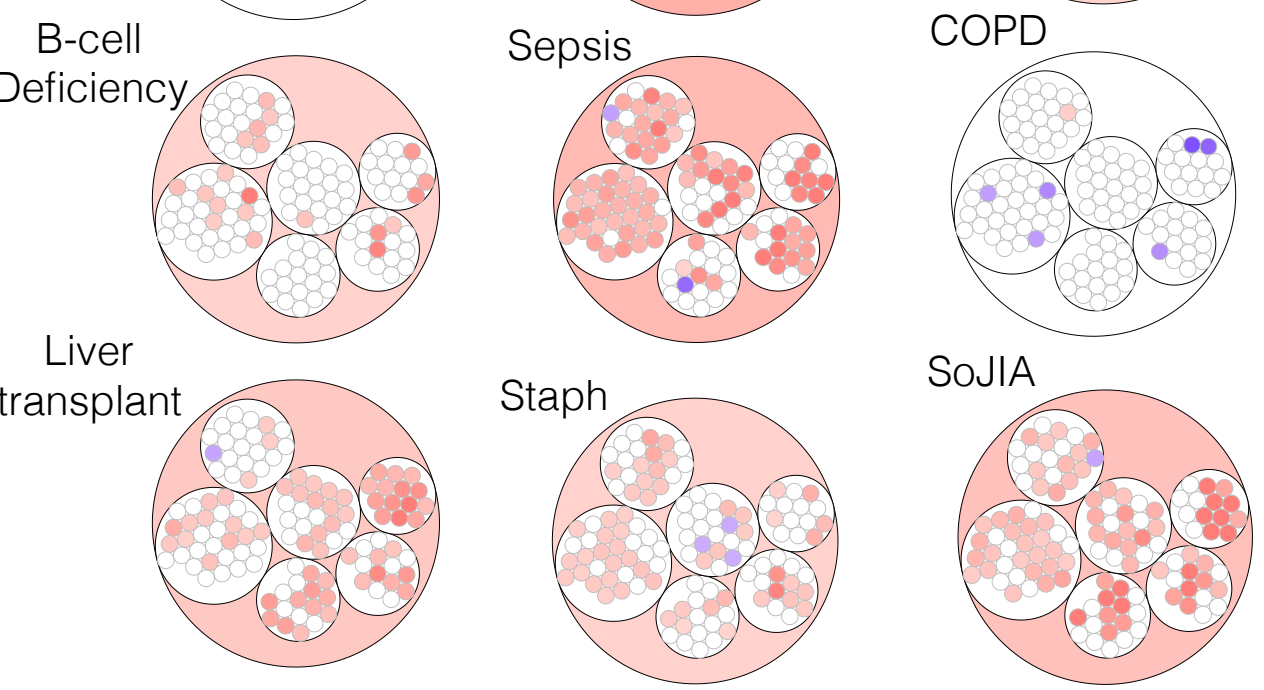

Figure S2: Patterns of changes in transcript abundance at the module and gene level for aggregate A28. The large circle plot represents the six modules constituting aggregate 28 , and the transcripts constituting each of the modules. Some genes on the Illumina BeadArrays can map to multiple probes, which explains the few instances where the same gene can be found in different modules. Patterns of changes in transcript abundance at the module-level and gene-level for aggregate A28 are shown for all 16 reference datasets. The position of the genes on each of these plots is fixed. Genes for which transcript abundance is changed are shown in red (increase) or in blue (decrease). 

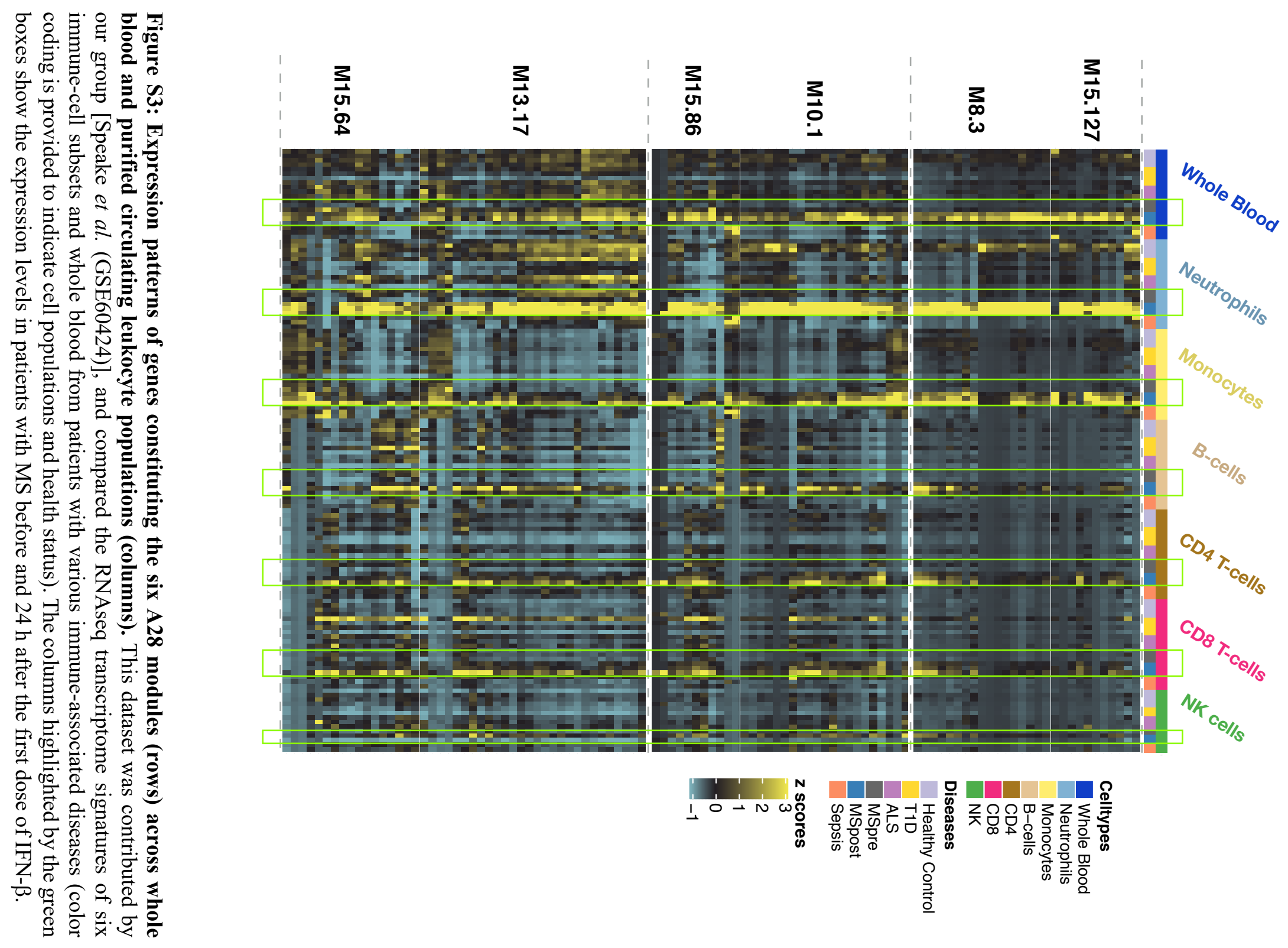

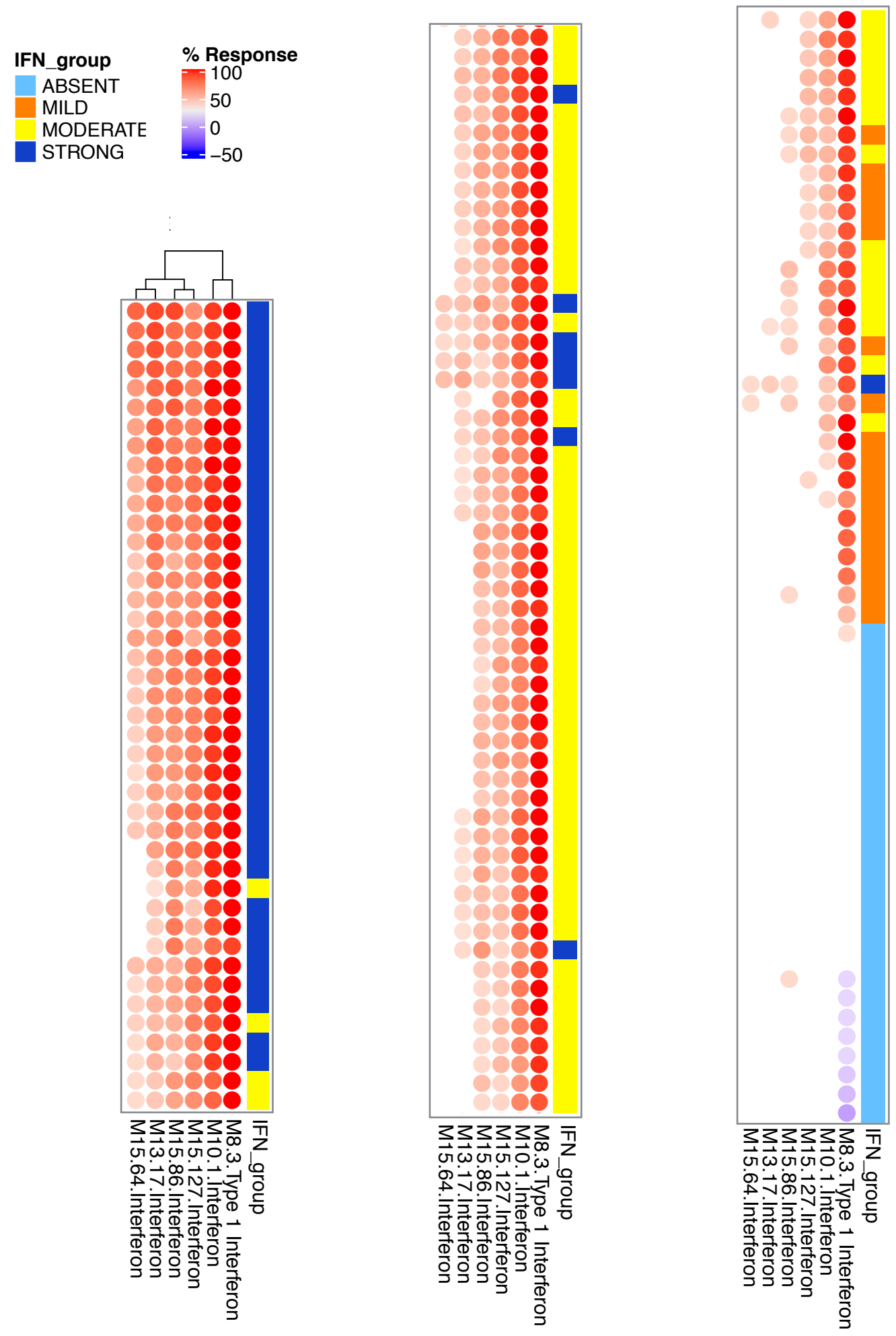

Figure S4: Abundance patterns of six interferon modules (A28) across individuals from an adult SLE cohort. The abundance levels for six interferon modules belonging to aggregate A28 (columns) is shown across a cohort of adult subjects diagnosed with SLE (rows). These subjects were part of the LUPUCE study: the dataset was contributed by our group [Chiche et al. (GEO ID GSE49454)] (11). Changes in transcript abundance were measured in reference to a cohort of healthy subjects included in the study. The colored label with interferon grouping information indicates the classification obtained by Chiche et al. based on three "second generation" modules. 


\section{Figures}

\section{Module repertoire construction}
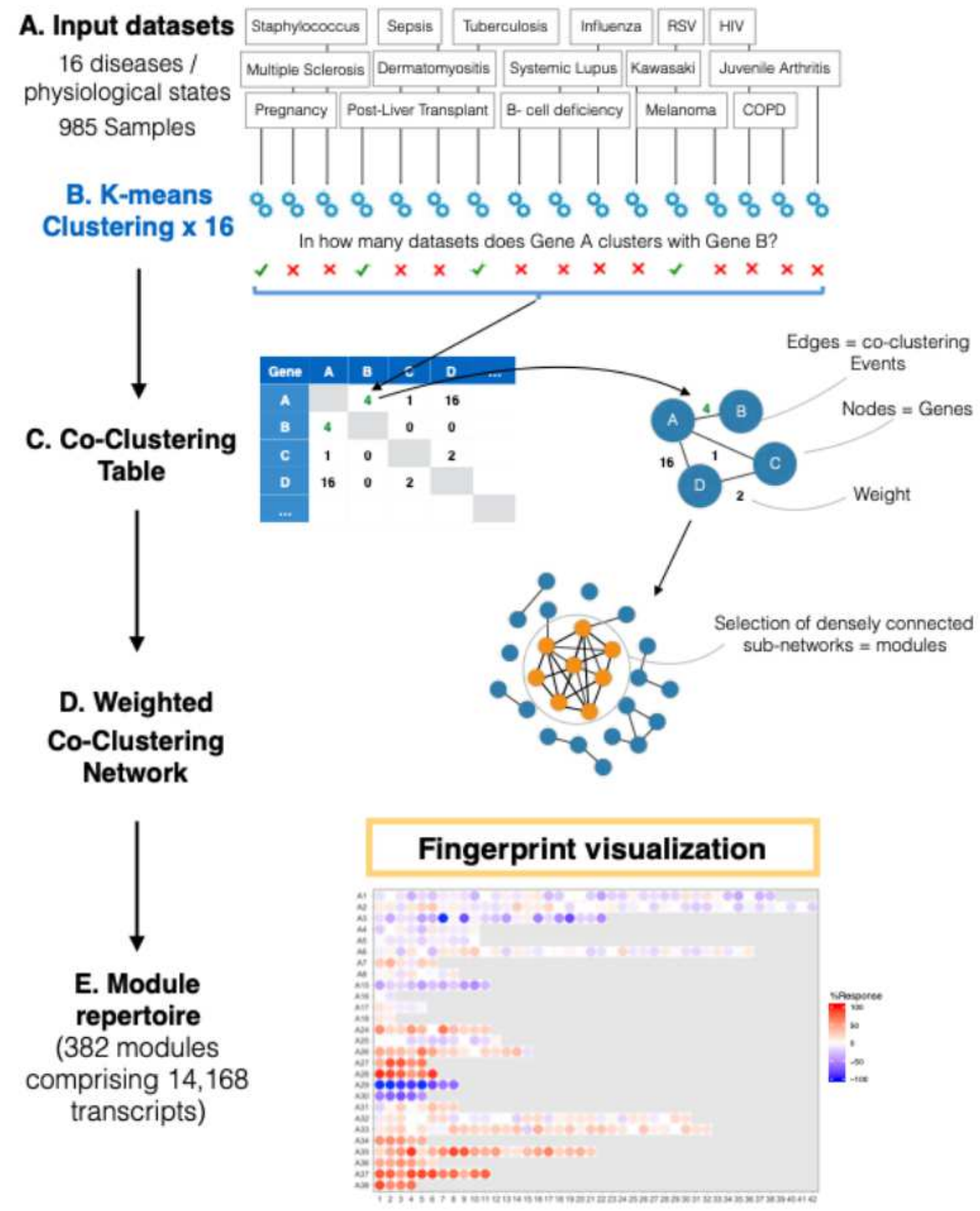

\section{Figure 1}

The module repertoire construction process. A. A collection of 16 transcriptome datasets spanning a wide range of immunological and physiological states were used as input. B. Each dataset was independently clustered via k-means clustering. C. Gene co-clustering events were recorded in a table, where the entries 
indicate the number of datasets in which co-clustering was observed for a given gene pair. D. The coclustering table served as the input to a weighted co-clustering graph (see also Supplementary Figure 1), where the nodes represent genes and the edges represent co-clustering events. E. The largest, most highly weighted sub-networks among a large network (here constituting 15,132 nodes) were identified mathematically and assigned a module ID. The genes constituting this module were removed from the selection pool and the process was repeated, resulting in the selection of 382 modules constituted by 14,168 transcripts.

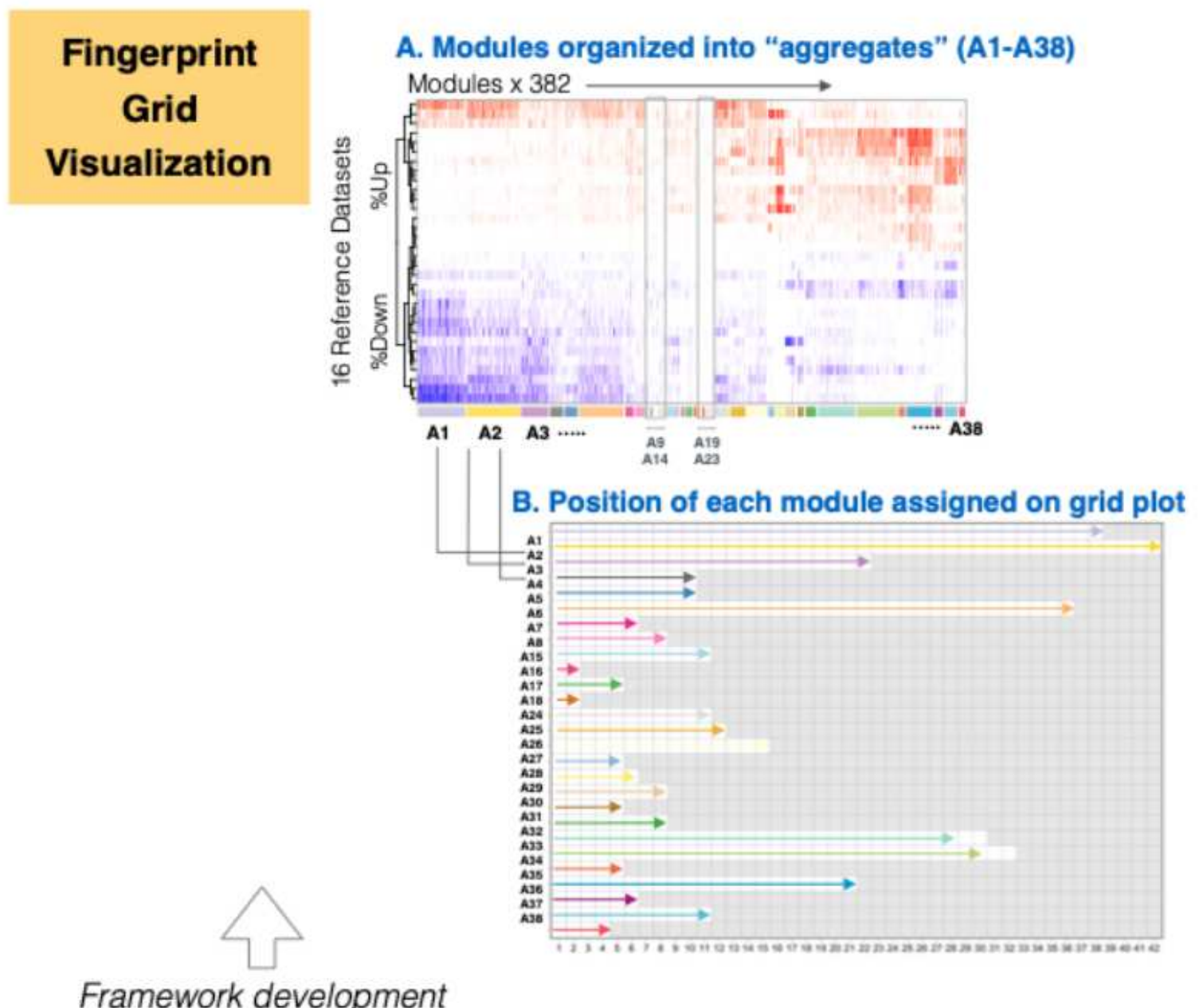

Framework development

Routine data analyses

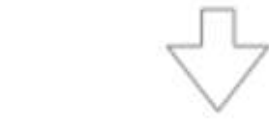

C. Changes in cases vs controls mapped

Controls Cases

VS.
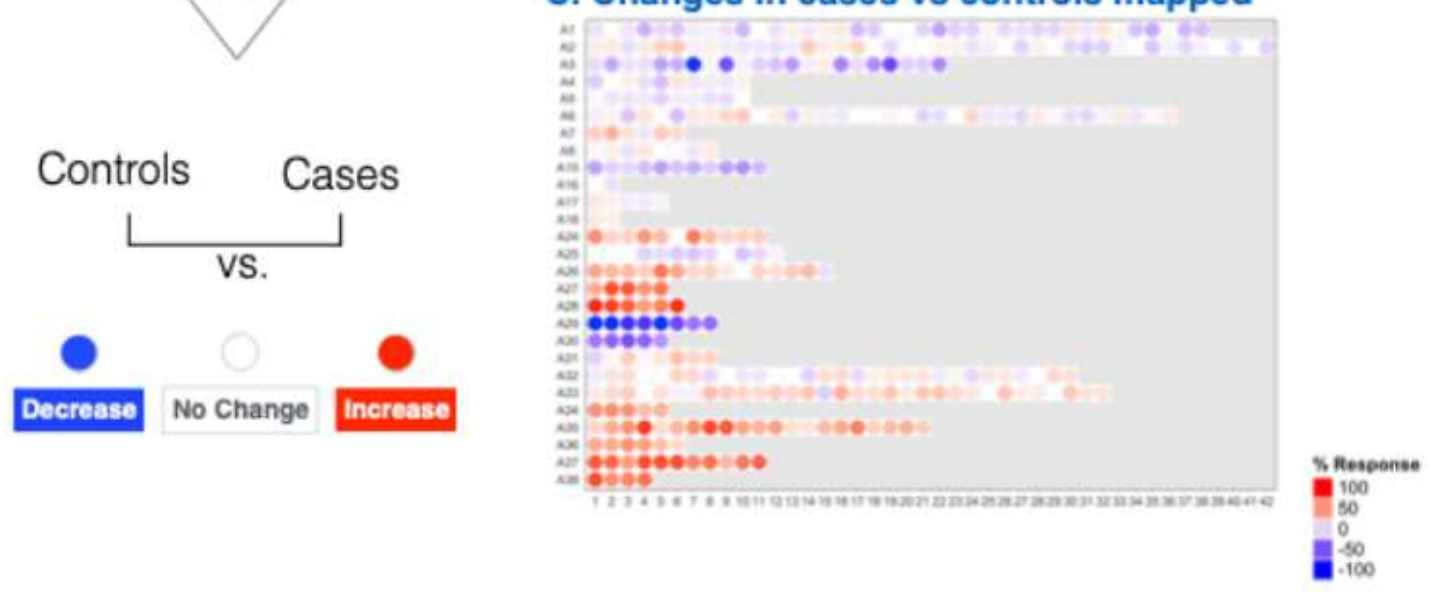


\section{Figure 2}

The development of module fingerprint grids. A. The modules were arranged onto the grid as follows: the master set of 382 modules was partitioned into 38 clusters (or aggregates) based on similarities among their module activity profiles across the sixteen reference datasets (A1-A38). B. A subset of 27 aggregates comprising 2 modules or more in turn occupied a line on the grid. The length of each line was adapted to accommodate the number of modules assigned to each cluster. The format of the grid was fixed for all analyses carried out using this modular framework. C. Changes in transcript abundance at the module level were mapped onto this grid and represented by color spots of varying intensity.

A. SLE patients $(\mathrm{N}=55)$ / Control subjects $(\mathrm{N}=14)$
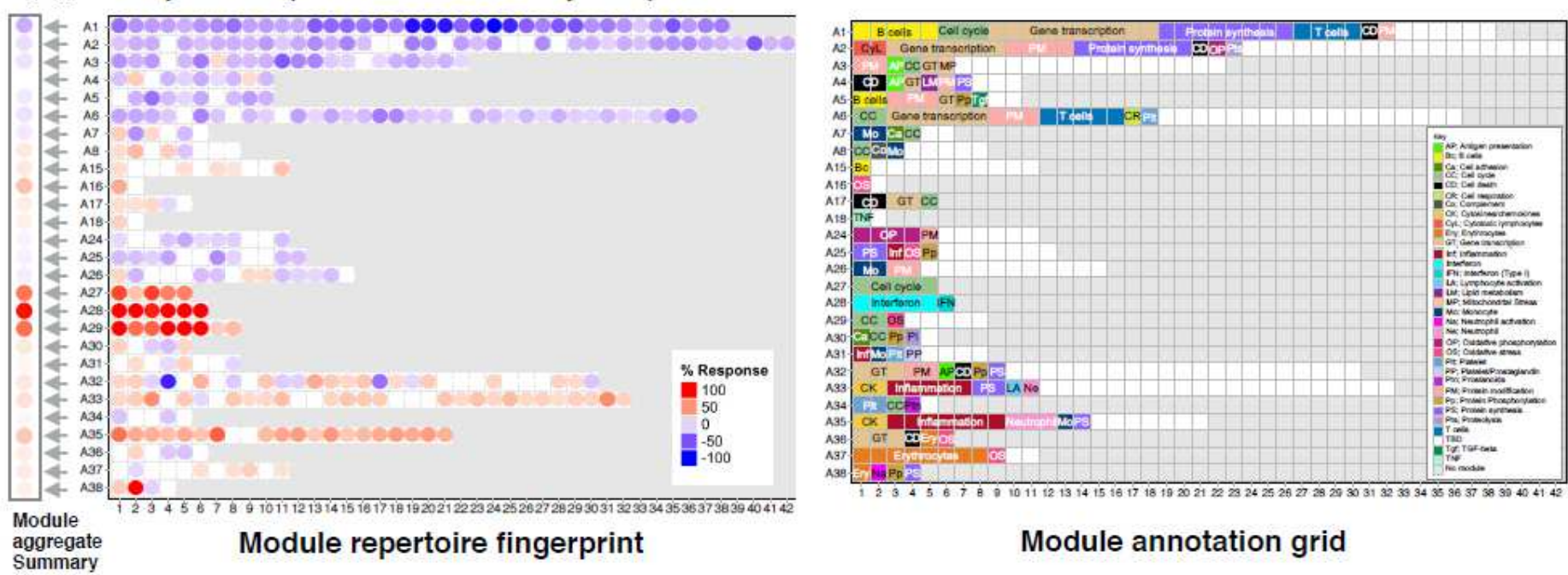

\section{Module annotation grid}

B.

MS

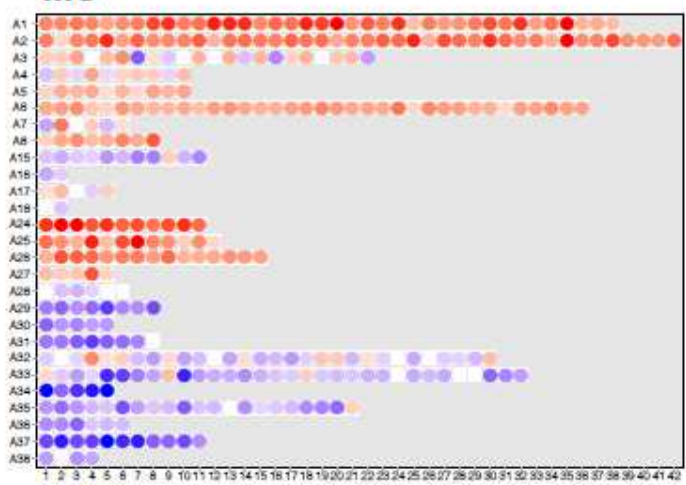

\section{S. aureus}

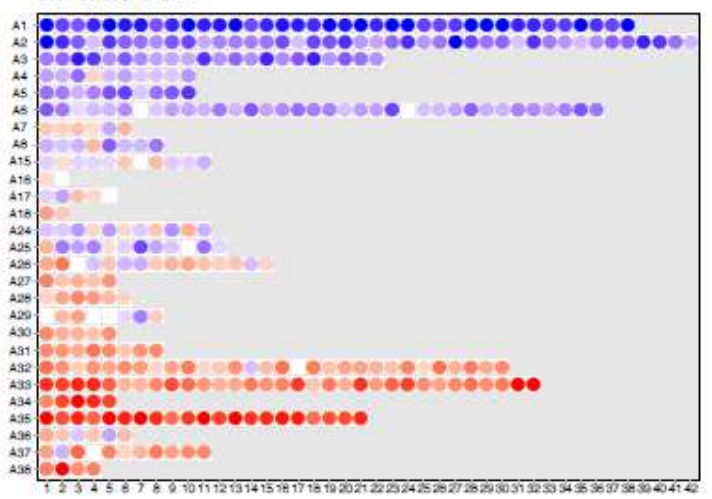

C. $\mathrm{COPD}$

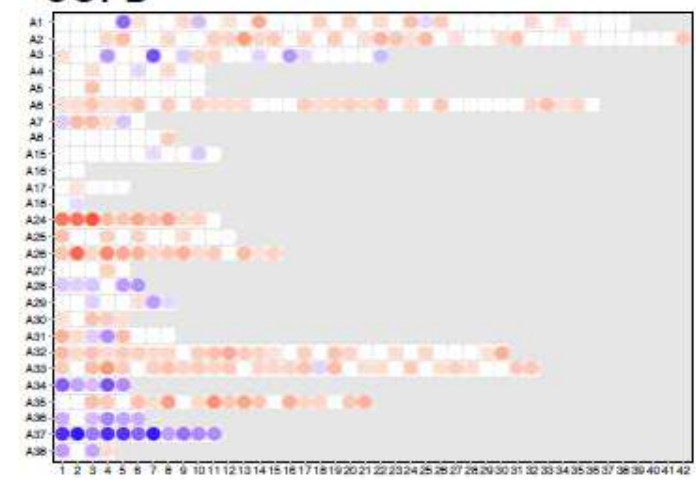

\section{Melanoma}

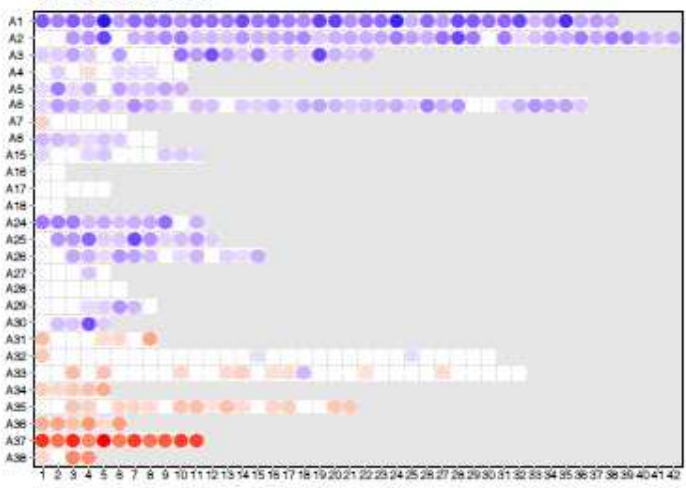




\section{Figure 3}

Fingerprint grid plots. A. Prototypical fingerprint grid plot: Changes in blood transcript abundance for patients with SLE compared to healthy controls are represented on a fingerprint grid plot for this illustrative use case. The modules occupy a fixed position on the fingerprint grid plots (see Figure 2). An increase in transcript abundance for a given module is represented by a red spot; a decrease in abundance is represented by a blue spot. Modules arranged on a given row belong to a module aggregate (here denoted as A1 to A38). Changes measured at the "aggregate-level" are represented by spots to the left of the grid next to the denomination for the corresponding aggregate. The colors and intensities of the spots are based on the average across each given row of modules. A module annotation grid is provided where a color key indicates the functional associations attributed to some of the modules on the grid (top right). Positions on the annotation grid occupied by modules for which no consensus annotation was attributed are colored white. Positions on the gird for which no modules have been assigned are colored grey. B-D. Fingerprint grid plots for additional reference datasets. 


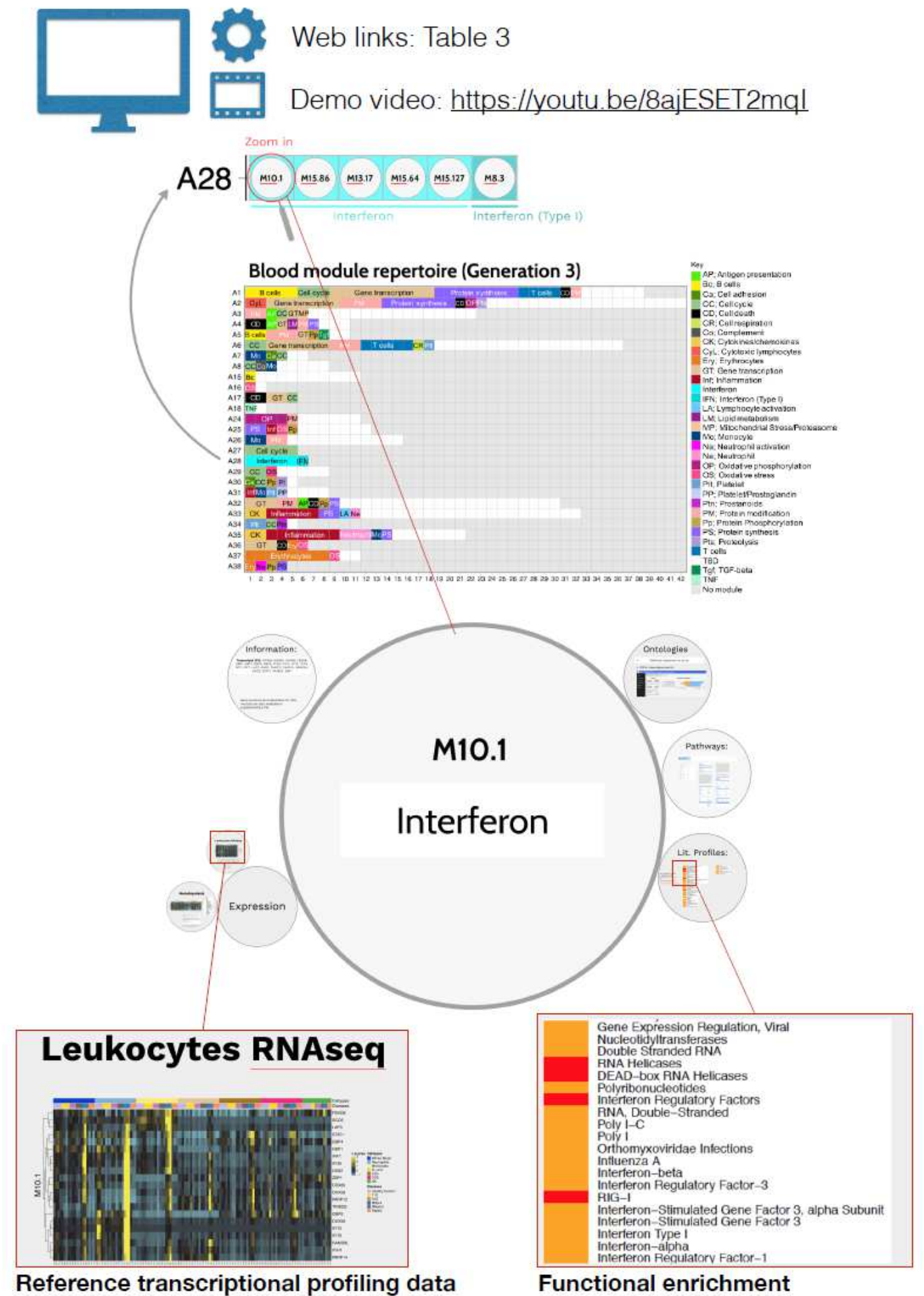

Figure 4

Functional annotation of the transcriptional module repertoire. An interactive application is available to explore the 382 modules comprising the blood transcriptome repertoire. A gene list, along with the ontology, pathway, literature term enrichment and transcriptional profiling data for reference transcriptome datasets (circulating leukocyte populations, hematopoiesis) is provided for each module. Zoom in and out functionalities for close-up examinations of the text and figures embedded in the 
presentation is possible. Web links providing access to modules within a given aggregate are listed in Table 3. For a demonstration video, please visit: https://youtu.be/8ajESET2mql.

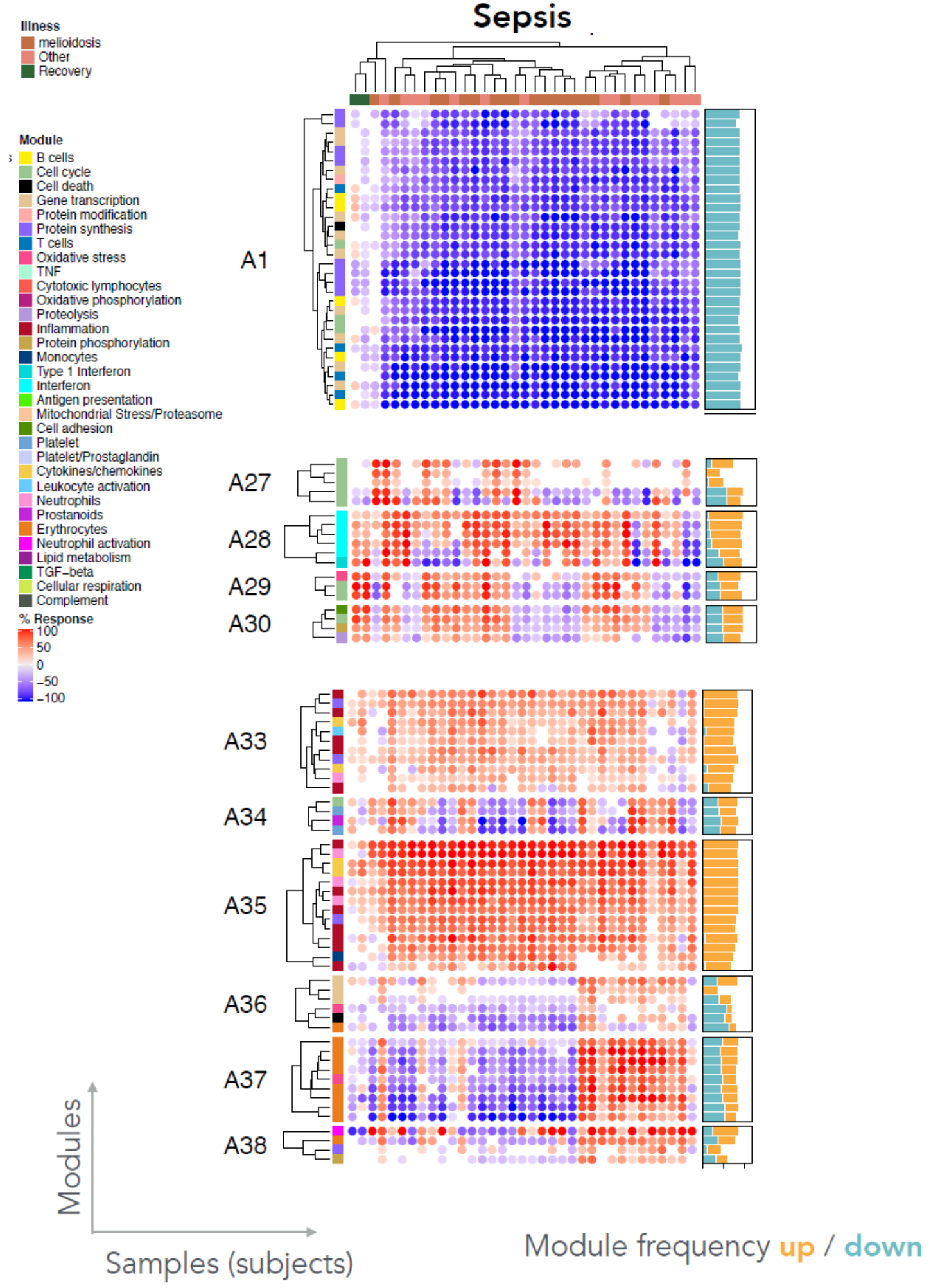

\section{Figure 5}

Individual-level module heatmap. Changes in transcript abundance were determined at the individual level across all modules constituting the repertoire. These changes are represented on a heatmap, where an increase in abundance of a given module is represented in red, and a decrease in abundance is 
represented in blue. The subjects are organized as columns and the modules as rows. The ordering on the heat map was determined by hierarchical clustering.

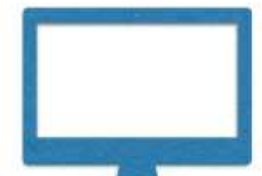

1

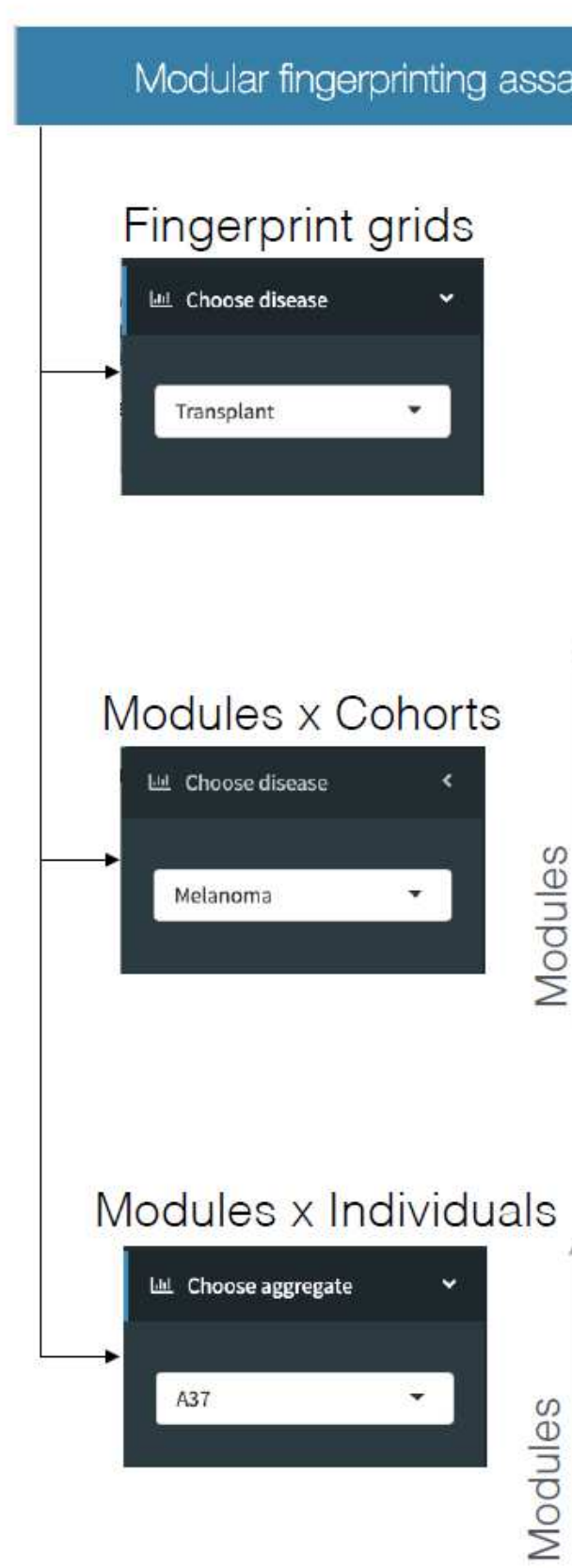

Shiny
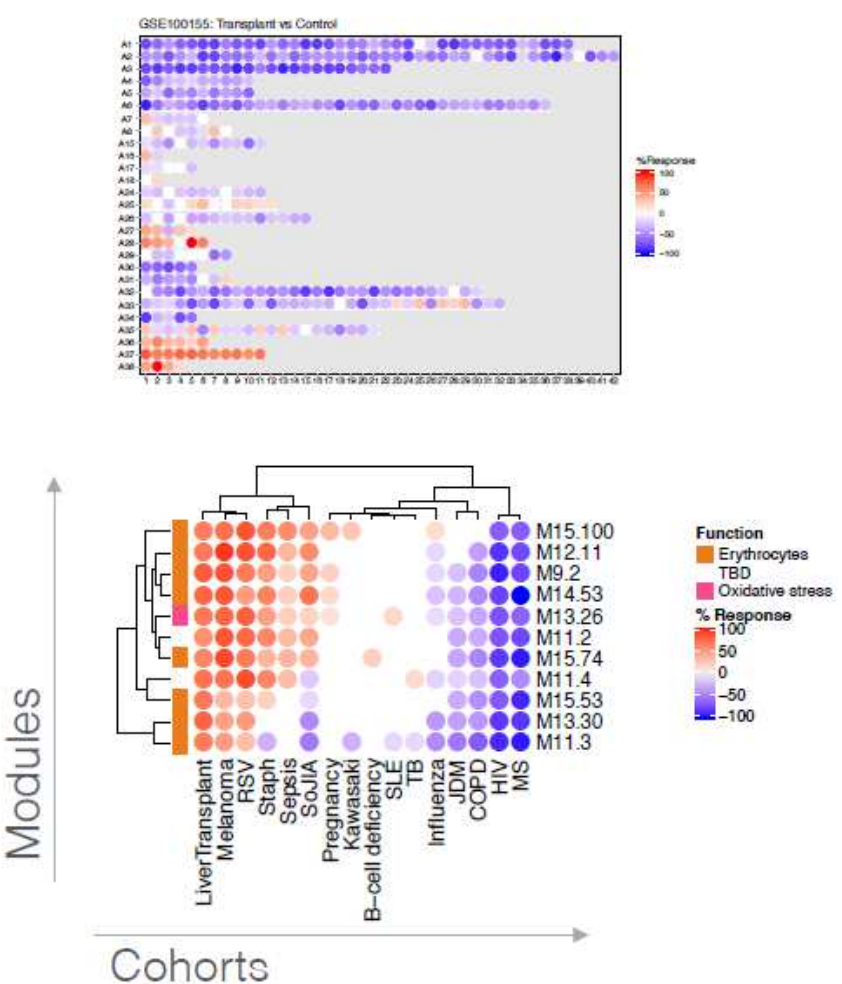

Cohorts

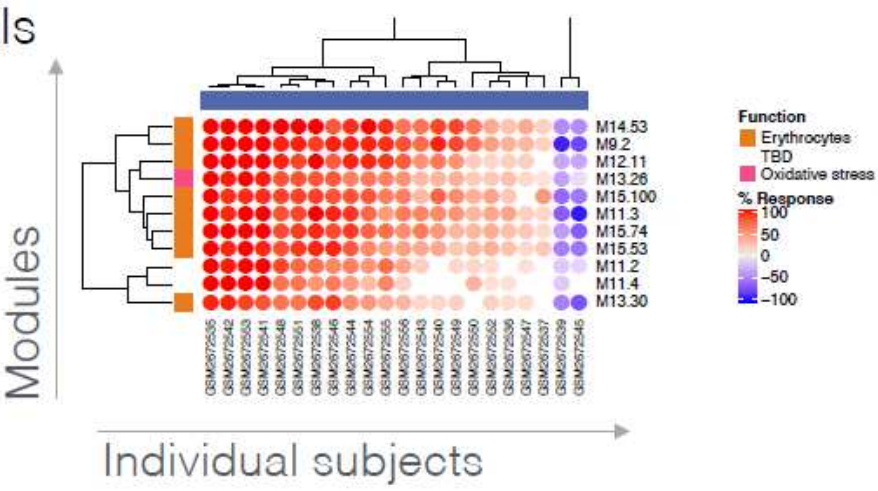

\section{Figure 6}

Web application to visualize multi-tiered module fingerprinting. An application was developed to explore the changes in transcript abundance at the module level across the 16 reference datasets used to construct the repertoire. Three types of plot can be displayed and exported: 1) fingerprint grids; 2) module 
heatmaps displaying changes in abundance in modules comprising a given aggregate across the 16 reference datasets; and 3) module heatmaps displaying changes in abundance in modules comprising a given aggregate across individuals constituting a given dataset. For accessing the application, please visit: https://drinchai.shinyapps.io/dc_gen3_module_analysis/\#. For a demonstration video, please visit: https://youtu.be/y__xKJo5e4.

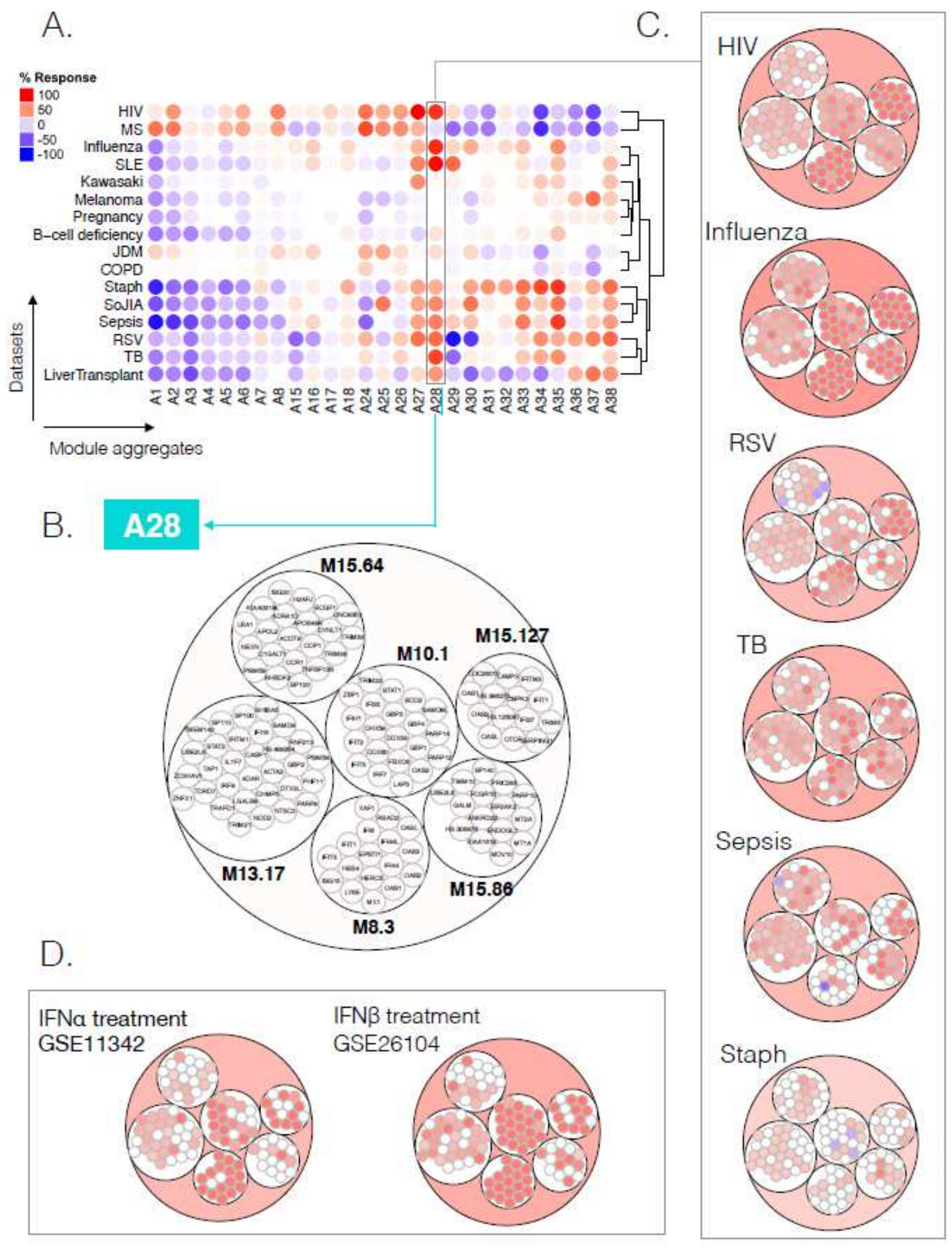

Figure 7 
Module aggregate abundance patterns across the 16 disease or physiological states. A. Patterns of changes in transcript abundance at the aggregate and cohort levels. Each column on the heat map corresponds to a "module aggregate", numbered A1 to A38. Modules A9- A14 and A19-A24 were excluded as they each only included one module. Each row on the heatmap corresponds to one of the 16 datasets used to construct the module framework. A red spot on the heatmap indicates an increase in abundance of transcripts comprising a given module cluster for a given disease or physiologic state. A blue spot indicates a decrease in abundance of transcripts. No color indicates no change. Disease or physiological states were arranged based on the level of similarity in the patterns of aggregate activity, determined via hierarchical clustering. B. Representation of the modules and genes constituting aggregate A28. The circle plot represents the six modules constituting aggregate 28 , and the transcripts constituting each of the modules. Some genes on the Illumina BeadArrays can map to multiple probes, which explains the few instances where the same gene can be found in different modules. C. Patterns of changes in hepatitis $C$ infection treated with IFNa [GSE11342 (16)] or patients with multiple sclerosis treated with IFN $\beta$ [GSE26104 (17)]. transcript abundance at the module-level and gene-level for aggregate A28. The circle plots illustrate the changes at the gene-level for this aggregate for $6 / 16$ datasets. The position of the genes on each of these plots is the same as shown in panel B. Genes for which transcript abundance is changed are shown in red (increase) or in blue (decrease). D. Patterns of changes in transcript abundance at the module and gene level for aggregate A28 in subjects treated with IFNa or IFN $\beta$ treatment The circle plots show changes in abundance for A28 transcripts in patients with 


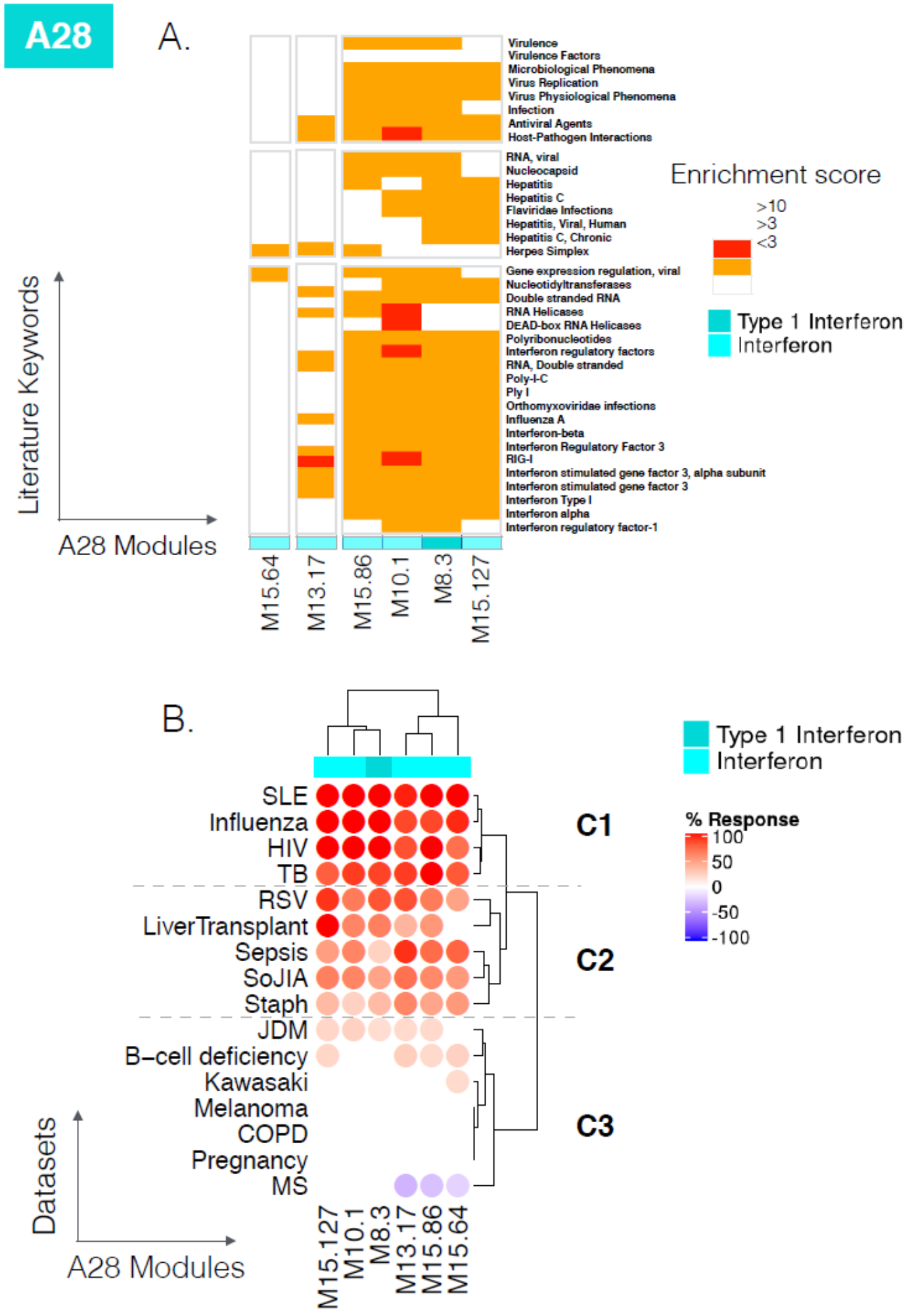

\section{Figure 8}

Literature profiles and patterns of changes in abundance across reference datasets for the modules comprising aggregate A28. A. Functional annotation by literature profiling: A portion of a heatmap comprising 382 modules organized as columns, and literature terms organized as rows. The six modules shown are associated with the consensus annotation "Interferon" or "Type 1 Interferon". The clusters of keywords associated with those modules are consistent with this annotation and provide added 
granularity to the module repertoire for functional profiling and interpretation. B. Changes in abundance across 16 reference datasets: the heatmap (top) represents the changes in abundance of transcripts constituting the six modules comprising aggregate A28 (columns). The modules are functionally associated with interferon responses. The 16 reference datasets are arranged as rows corresponding to different health states. The columns and rows were arranged by hierarchical clustering. Such heatmaps can be accessed and exported for all 16 datasets and 38 module aggregates using the web application: https://drinchai.shinyapps.io/dc_gen3_module_analysis/\# (under the "MODULES x STUDIES" tab).
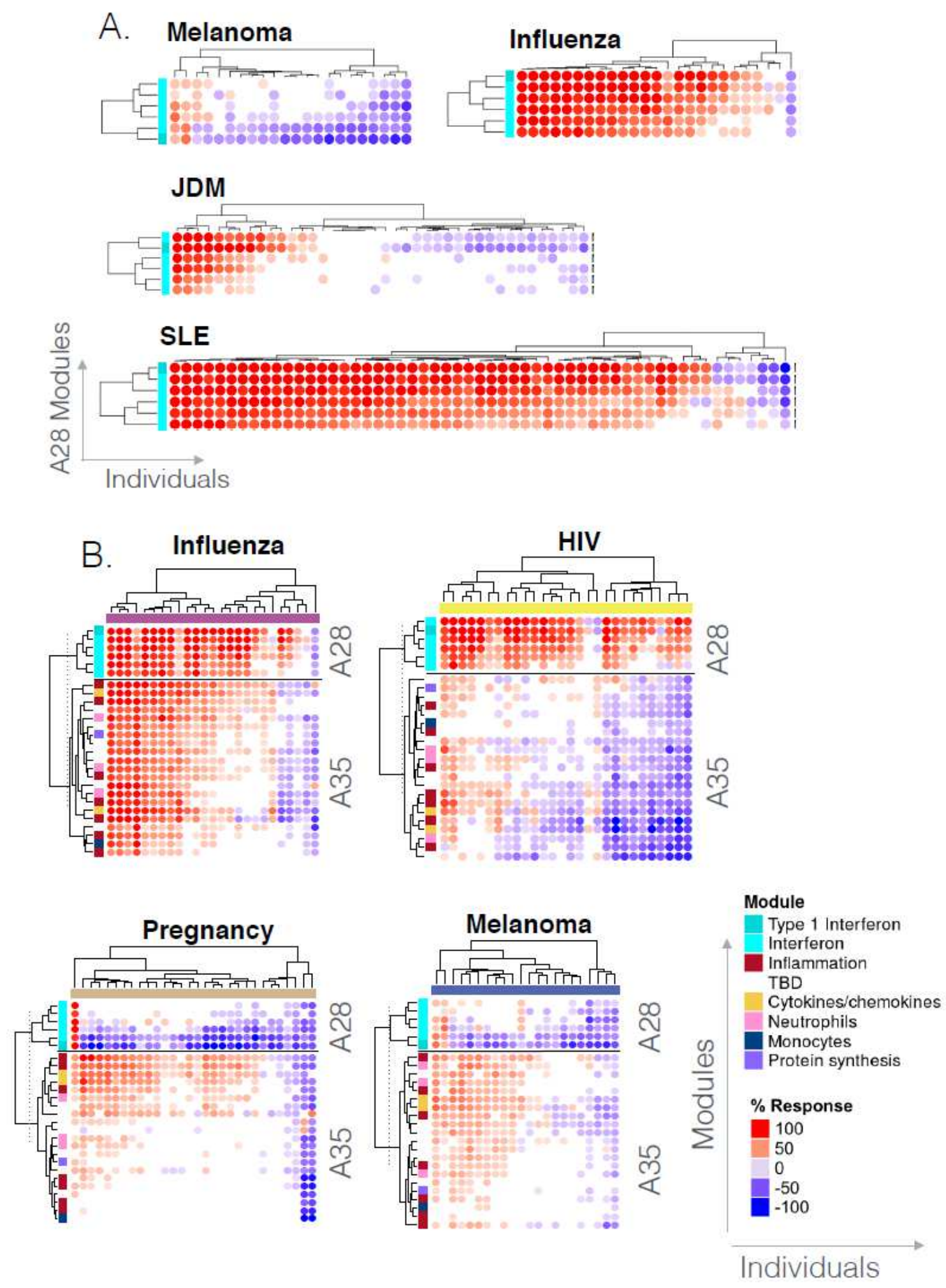


\section{Figure 9}

Abundance patterns across individuals. A. Changes in abundance for A28 modules. The heatmaps display the changes in abundance for the same six modules (rows) across individuals (columns) in four reference cohorts. The rows and columns on the heatmap are arranged based on similarities in abundance patterns. B. Changes in abundance for A28 and A35 modules. The heatmaps display the changes in abundance of six modules constituting aggregate A28 and 21 modules constituting aggregate A35 (rows) across individuals (columns) in four reference datasets. Functional annotations associated with different modules is indicated by a color code and corresponding legend. Such heatmaps can be accessed and exported for all 16 datasets and 38 module aggregates using the web application: https://drinchai.shinyapps.io/dc_gen3_module_analysis/\# (under the "MODULES x INDIVIDUALS" tab). 